\title{
Construction of a Redox-Related Prognostic Model with Predictive Value in Survival and Therapeutic Response for Patients with Lung Adenocarcinoma
}

\author{
Lingyan Xiao $\mathbb{D}^{1},{ }^{1}$ Qian Li $\mathbb{D},{ }^{2}$ Yongbiao Huang $\mathbb{D}^{1},{ }^{1}$ Zhijie Fan $\mathbb{D}^{1},{ }^{1}$ Li Ma $\mathbb{D}^{1},{ }^{1}$ Bo Liu $\mathbb{D}$, ${ }^{1}$ \\ and Xianglin Yuan iD 1 \\ ${ }^{1}$ Department of Oncology, Tongji Hospital, Tongji Medical College, Huazhong University of Science and Technology, \\ Wuhan, China \\ ${ }^{2}$ Department of Pathophysiology, School of Basic Medicine, Tongji Medical College, \\ Huazhong University of Science and Technology, Wuhan, China \\ Correspondence should be addressed to Bo Liu; boliu888@hotmail.com and Xianglin Yuan; yuanxianglin@hust.edu.cn
}

Received 15 September 2021; Revised 27 December 2021; Accepted 18 January 2022; Published 25 February 2022

Academic Editor: Kathiravan Srinivasan

Copyright ( $\odot 2022$ Lingyan Xiao et al. This is an open access article distributed under the Creative Commons Attribution License, which permits unrestricted use, distribution, and reproduction in any medium, provided the original work is properly cited.

Background. Lung adenocarcinoma (LUAD) represents the most common histological subtype of lung cancer. Redox plays a significant role in oncogenesis and antitumor immunity. In this study, we aimed to investigate the prognostic redox-associated genes and construct a redox-based prognostic signature for LUAD. Materials and Methods. A discovery cohort containing 479 LUAD samples from The Cancer Genome Atlas (TCGA) was analyzed. We identified prognostic redox-associated genes by weighted correlation network analysis (WGCNA) and univariate Cox regression analysis to construct a prognostic model via least absolute shrinkage and selection operator (LASSO)-multivariate Cox regression analyses. The performance of the redox-based model was validated in the TCGA cohort and an independent cohort of 456 samples by Cox regression analyses, log-rank test, and receiver operating characteristic (ROC) curves. Correlations of the model with clinicopathological variables and lymphocyte infiltration were assessed. Gene set enrichment analysis (GSEA) was used to clarify the underlying mechanism of the prognostic model. We constructed a nomogram based on the model and created calibration curves to show the accordance between actual survival and predicted survival of the nomogram. Results. Stepwise analyses identified 6 prognostic redox-associated genes of LUAD and constructed a prognostic model that performed well in both the discovery and validation cohorts. The model was found to be associated with tumor stage, mutation of TP53 and EGFR, and lymphocyte infiltration. The model was mainly involved in the regulation of the cell cycle, DNA replication and repair, NADH metabolism, and the p53 signaling pathway. Calibration curves showed the high predictive accuracy of the nomogram. Conclusions. This study explored the role of redoxassociated genes in LUAD and constructed a prognostic model of LUAD. The signature was also associated with tumor progression and therapeutic response to immunotherapy. These findings contributed to uncovering the underlying mechanism and discovering novel prognostic predictor of LUAD.

\section{Introduction}

Comprising $40 \%$ of lung cancer cases, lung adenocarcinoma has occupied a core position in lung cancer due to its high mortality and morbidity. Although the 5-year survival rate has reached approximately $60 \%$ for patients with early-stage LUAD, the number declines to $25 \%$ when all stages are combined [1].
The prediction of survival for patients with LUAD can aid in tailoring optimal treatment strategies. The TNM staging system remains the most frequently used indicator to predict outcome for patients with LUAD. Targeted therapy and immunotherapy represent emerging and effective therapies for LUAD. For patients in the advanced stage without targeted mutations, immune checkpoint inhibitor (ICI) therapy may demonstrate satisfactory efficacy. With 
the rise of various treatment strategies, more reliable prognostic biomarkers are needed to achieve individual therapy.

Reactive oxygen species (ROS) are oxygen (O)-containing molecules generated mainly by oxidative phosphorylation in mitochondria and NADPH oxidases (NOXs). ROS are involved in various biological processes due to their high reactivity toward proteins, nucleic acids, and lipids [2]. ROS can affect the function of proteins by modifying cysteine residues, giving rise to diseases such as asthma, diabetes, and cancer [3-6]. Redox homeostasis refers to the balance between the generation and elimination of ROS [7]. Upsetting this delicate redox balance was related to the initiation and progression of cancer [8-10]. Oncogenesis and metastasis can be inhibited by high ROS concentration, and cancer cells can promote the synthesis of antioxidants to buffer ROS to avoid this inhibition [11]. Pathways that are essential to oncogenesis, such as PI3K/AKT, MAPK, and $\mathrm{NF}-\kappa \mathrm{B}$, are regulated by redox [12-15]. Oncogenes and tumor suppressor genes such as TP53, PTEN, and RAS can also interact with the redox system to determine the fate of cells [2, 16-20]. Cancer stem cells (CSCs) have been reported to have unique redox profiles with low ROS level facilitating the stemness properties and drug resistance of CSCs, and the ability of cancer stem cells to generate tumorspheres can be limited by ROS [21].

Given the great significance of redox in tumorigenesis, we hypothesized that redox-associated genes may have prognostic value in LUAD. We conducted multiple analyses to identify the significant prognostic redox-associated genes based on expression data of LUAD acquired from TCGA. A prognostic model was constructed based on prognostic redox-associated genes by LASSO and multivariate Cox regression analyses, which could predict the survival outcome and immunophenotypes of patients with LUAD.

\section{Materials and Methods}

\subsection{Data Collection and Differential Expression Analysis.} Expression data, mutation data, and corresponding clinical information of LUAD were downloaded from the TCGA database. A total of 594 samples were analyzed (N (nor$\mathrm{mal})=59, \mathrm{~N}($ tumor $)=535)$, among which 522 samples had clinical information. Microarray data used for validation were acquired from the Gene Expression Omnibus (GEO) database (GSE32863, GSE43458, GSE37745, GSE31210, and GSE50081). A total of 4338 redox-associated genes were collected from the GSEA website (https://www.gsea-msigdb. org), the Gene Ontology website (http://geneontology.org/), OMIM database (https://omim.org/), and GeneCard database (https://www.genecards.org/). The package "edgeR" was used to remove the genes with low expression, normalize the expression data, and identify differentially expressed redox-associated genes. First, we conducted differential expression analysis between the total tumor and normal samples $(N=594)$. Next, the differential expression analysis between the paired tumor and peritumoral tissues $(N=114)$ was performed to eliminate the effect caused by the huge difference in the total sample number between the tumor and normal tissues. Genes meeting the filtering criteria of false discovery rate $(F D R)<0.05$ and $\mid \log 2$ fold change $(\mathrm{FC}) \mid>2.0$ in two differential analyses were considered differentially expressed genes.

2.2. GO and KEGG Analyses. Gene Ontology (GO) enrichment and Kyoto Encyclopedia of Genes and Genomes (KEGG) pathway analyses were conducted using the $R$ package "clusterProfiler" to clarify the functions of the dysregulated redox-associated genes. Selection criteria are both $\mathrm{q}$ value and $p$ value $<0.05$.

2.3. Establishing a Prognostic Model. WGCNA is a powerful bioinformatic tool for identifying co-expressed genes with similar biological functions to generate co-expression modules and correlating co-expression modules with parameters of interest [22]. Thus, WGCNA has been frequently applied in identifying biomarkers that are associated with specific biological functions or clinical characteristics [23-25]. In our study, WGCNA was used to extract significant gene modules associated with survival and clinical variables, including age, gender, and stage, using the "WGCNA" package. Next, univariate Cox regression analysis and log-rank test were performed for genes in the survival-related gene module to further identify prognostic redox-associated genes. LASSO and multivariate Cox regression analyses were conducted using the "glmnet" package to determine the prognostic genes for model construction, and the coefficients obtained in multivariate Cox regression were set as weights.

2.4. Verification of the Prognostic Model. Differential expression of the prognostic redox-associated genes in the model was verified in GSM43458 $(N=110)$ and GSM32863 $(N=116)$ using the "limma" package. Survival curves of these genes were obtained from Gene Expression Profiling Interactive Analysis (GEPIA) (http://gepia.cancer-pku.cn/). After removing the samples without complete survival and clinical data, GSE37745 $(N=196)$, GSE31210 $(N=79)$, and GSE50081 $(N=181)$ were merged into the GEO cohort $(N=456)$. The prognostic performance of the model was assessed in the TCGA and GEO cohorts. The Kaplan-Meier survival curves were plotted to compare the overall survival (OS) of the high- and low-risk groups. The correlation of the prognostic model with survival was indicated by the univariate Cox regression analysis. Then, the multivariate Cox regression analysis was performed to evaluate whether the risk score could affect the survival of patients with LUAD independently. The "survival" package was used to conduct the survival analyses. The predictive power of the prognostic model was assessed by ROC curves using the "survivalROC" package.

2.5. Relationship between the Prognostic Model and Clinical Parameters and Gene Set Enrichment Analysis. The distribution of the risk score in patients divided by clinical variables was compared to show the correlation between risk 
score and cancer progression. The mutation rate of driver genes was compared between the high- and low-risk groups based on the chi-square test. GSEA was performed to reveal the involved GO terms and pathways of our prognostic model based on filter criteria of NOM $p$ value $<0.01$ and FDR q value $<0.05$.

2.6. Association of the Prognostic Model With Infiltration of Lymphocytes. Estimation of STromal and Immune cells in MAlignant Tumours using Expression data (ESTIMATE), an algorithm that can work out the proportion of stromal cells and immune cells [26], was applied to obtain the immune score and stromal score, which represented the abundance of immune cells and stromal cells, respectively. Major immune cells in the tumor microenvironment of the TCGA cohort were downloaded from TIMER [27, 28] (http://timer.comp-genomics.org). Cell-type Identification By Estimating Relative Subsets Of RNA Transcripts (CIBERSORT) is a computational method that uses a deconvolution algorithm to qualify the composition of cells based on expression data. Here, the CIBERSORT algorithm was run by $R$ language to further show the proportion of tumor-infiltrating lymphocyte subsets [29]. The number of permutations was set to 1000 .

2.7. Construction and Validation of a Nomogram. A nomogram based on the prognostic model was established using the "rms" package to estimate the survival probability of patients. To show the concordance between predicted survival and actual survival, the calibration curves were created for both the TCGA and GEO cohorts.

2.8. Statistical Analysis. All statistical analyses were carried out with $R$ software 4.0.0. GSEA was conducted by the GSEA software version 4.1.0. The $p$ value was corrected using the Benjamini-Hochberg approach in differential expression analysis between the normal and tumor tissues. Survival differences were evaluated by the log-rank test. Differences in gene expression and immunophenotypes between the high- and low-risk groups were assessed by the Wilcoxon test, and gene mutation rates between the two groups were compared by the chi-square test. $P<0.05$ was considered statistically significant.

\section{Results}

3.1.Differentially Expressed Redox-Associated Genes of LUAD. Figure 1 presents the flowchart of our research. A total of 366 and 331 dysregulated redox-associated genes meeting the cutoff value of $\mid \log 2$ fold change (FC) $\mid>2.0$ and adjusted $p$ value $(\mathrm{FDR})<0.05$ were identified in the differential expression analysis of overall samples and paired samples, respectively (Figures 2(a) and 2(b)). A total of 290 overlapping dysregulated redox-associated genes were identified from these two differential expression analyses, including 75 downregulated genes and 215 upregulated genes (Figure 2(c)).
3.2. Functions of Dysregulated Redox-Associated Genes. The dysregulated redox-associated genes participate in the response to oxidative stress, NADP activity, hormone metabolism, DNA packaging, and oxygen binding (Figures 3(a) and 3(c)). The dysregulated genes are mainly involved in alcoholism, neutrophil extracellular trap formation, systemic lupus erythematosus, IL-17 signaling pathway, and transcriptional misregulation in cancer (Figures $3(\mathrm{~b})$ and $3(d))$. The response to oxidative stress is the most significantly activated function of these dysregulated redox-associated genes, with almost $20 \%$ of the dysregulated redox-associated genes participating in this function.

3.3. Establishment of a Prognostic Model. Two gene modules (MEblue and MEturquoise) were found to correlate with the survival of LUAD patients in WGCNA (Figure 4(a)). The MEturquoise module was selected for further analyses since it correlated with not only survival but also clinical parameters, including stage, age, and gender. Then, log-rank test and univariate Cox regression analysis identified 36 prognostic genes from 145 redox-associated genes in the MEturquoise module (Figure 4(b)). Ten prognostic genes (AHNAK2, IGF2BP1, CDC25 C, ABCC2, CPS1, CDX2, NTSR1, SLC2A1, ARNTL2, and SLC7A5) were identified by the LASSO regression (Figure 4(c)). Next, to avoid underestimation of coefficients and minimize variables, the multivariate Cox regression was performed to select 6 prognostic genes (AHNAK2, CDC25 C, CPS1, CDX2, NTSR1, and SLC2A1) to construct a prognostic model (Figure 4(d), Supplementary Table 1). All six prognostic genes were oncogenes adversely affecting survival and were statistically significant in the multivariate Cox regression analyses except NTSR1. The risk score can be calculated by adding together the products of the expression level of genes and corresponding coefficients for each patient (Supplementary Table 1). Patients in the TCGA and GEO cohorts were classified into a high-risk group and a low-risk group by the cutoff value of the median risk score. The clinical characteristics of the discovery cohort and validation cohort are shown in Table 1 and Supplementary Table 2.

3.4. The Prognostic Model Had Robust Prognostic Performance. Six prognostic redox-associated genes were differentially expressed in GSE36823 (Supplementary Figure 1(a)) and GSE43458 (Supplementary Figure 1(b)). The results of survival curves in GEPIA were also consistent with our findings. AHNAK2 $(\mathrm{HR}=1.5, p$ value $=0.0088)$, CDC25 C $(\mathrm{HR}=2.5, p$ value $<0.001), C P S 1 \quad(\mathrm{HR}=1.5$, $p$ value $=0.052), \mathrm{CDX} 2(\mathrm{HR}=1.6, p$ value $=0.011)$, NTSR 1 $(\mathrm{HR}=1.6, p$ value $=0.0035)$, and SLC2A1 $(\mathrm{HR}=1.6, p$ value $<0.001$ ) are unfavorable indicators of survival (Supplementary Figure 2(a)-(f)). In addition, AHNAK2 ( $\mathrm{HR}=1.7, p$ value $=0.017), C D C 25 C(\mathrm{HR}=1.9, p$ value $=0.0039), C P S 1$ $(\mathrm{HR}=1.6, p$ value $=0.028), \quad$ NTSR $1 \quad(\mathrm{HR}=1.7, p$ value $=0.038)$, and SLC2A1 $(\mathrm{HR}=1.6, p$ value $=0.028)$ ad versely affected disease-free survival (DFS) (Supplementary Figures 2(g), (h), (i), (k), and (l)), whereas no obvious difference in DFS was observed between the low-CDX2 and high-CDX2 groups (Supplementary Figure 2(j)). 


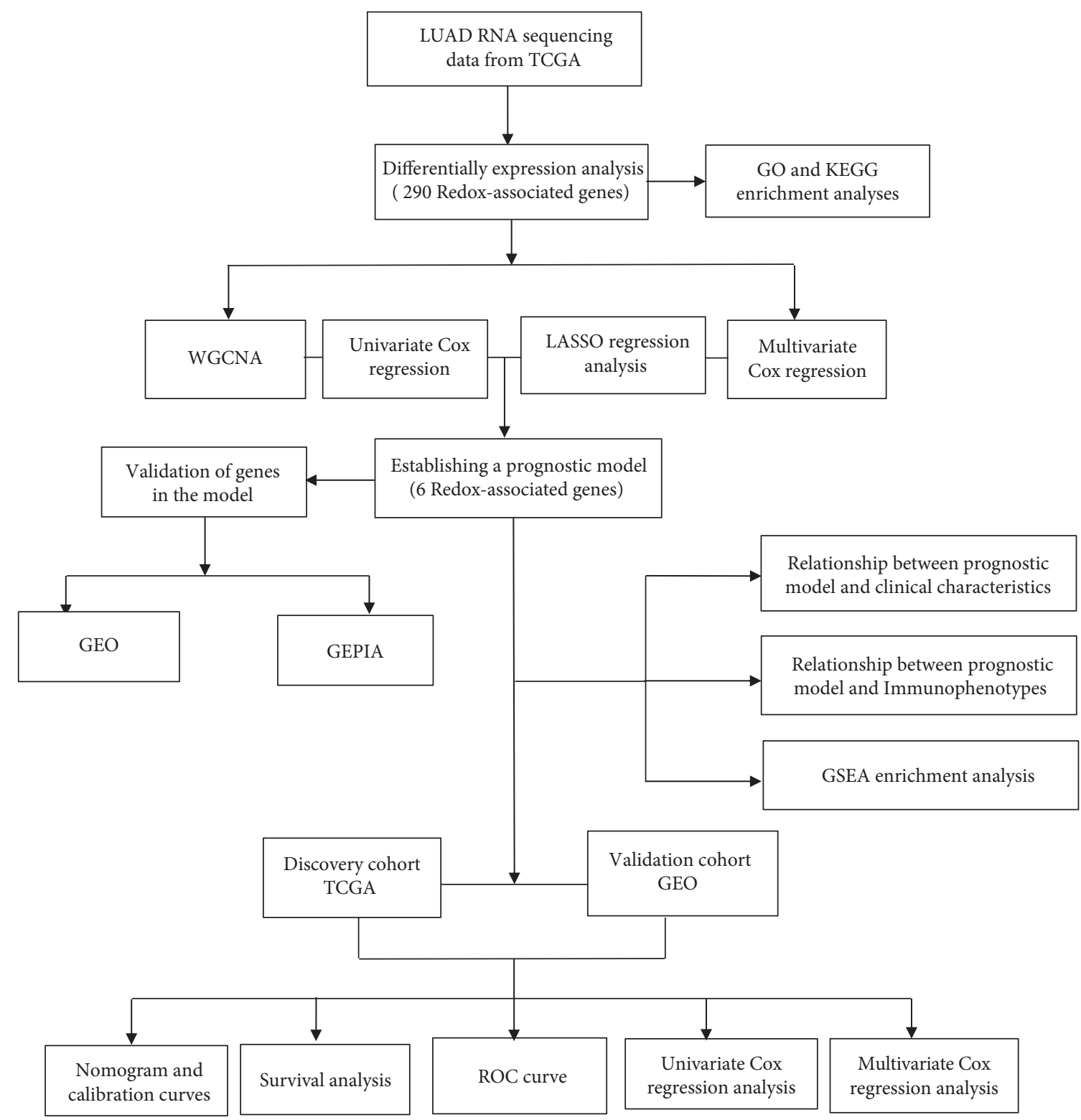

Figure 1: Flowchart of this study.

After verifying the differential expression and prognostic value of the genes in the model, the performance of the prognostic model was confirmed in the discovery (TCGA) and validation cohorts (GSE37745, GSE31210, and GSE50081). By survival curves and univariate Cox regression analysis, we identified the risk score as an unfavorable indicator of survival in both the TCGA $(\mathrm{HR}=1.233, p$ value $<0.001)$ (Figures 5(a) and 5(c)) and GEO cohorts $(\mathrm{HR}=1.222$, $p$ value $=0.003)$ (Figures $5(\mathrm{~b})$ and $5(\mathrm{~d})$ ). The results of multivariate Cox regression analysis demonstrated that the prognostic value of our model was not affected by clinicopathological parameters, including age, gender, and stage, in the TCGA $(\mathrm{HR}=1.23, p$ value $<0.001$ ) (Figure $5(\mathrm{e})$ ) and GEO cohorts $(\mathrm{HR}=1.188$, $p$ value $=0.012)$ (Figure 5(f)). The AUCs for 1-, 3-, and 5year survival in the TCGA cohort (Figure 5(g)) were 0.709, 0.705, and 0.635, respectively. The AUCs for 1-, 3-, and 5-year survival in the GEO cohort (Figure 5(h)) were 0.617, 0.633 , and 0.612 , respectively.

\subsection{Risk Score Was Associated with Tumor Stage and Driver} Gene Mutation. A statistically significant difference in the distribution of risk score between patients stratified by clinicopathological status was observed. Although no association was observed between age and risk score (Figure 6(a)), the risk score was associated with gender, stage, lymph node metastasis, and distant metastasis (Figures 6(b)-6(f)). The risk score was higher in male patients and patients with advanced tumor, suggesting that the prognostic model was related to tumor progression and clinical outcome. Based on analysis of mutation data in TCGA, we found that the low-risk group and the high-risk group had significantly different gene mutation frequencies 


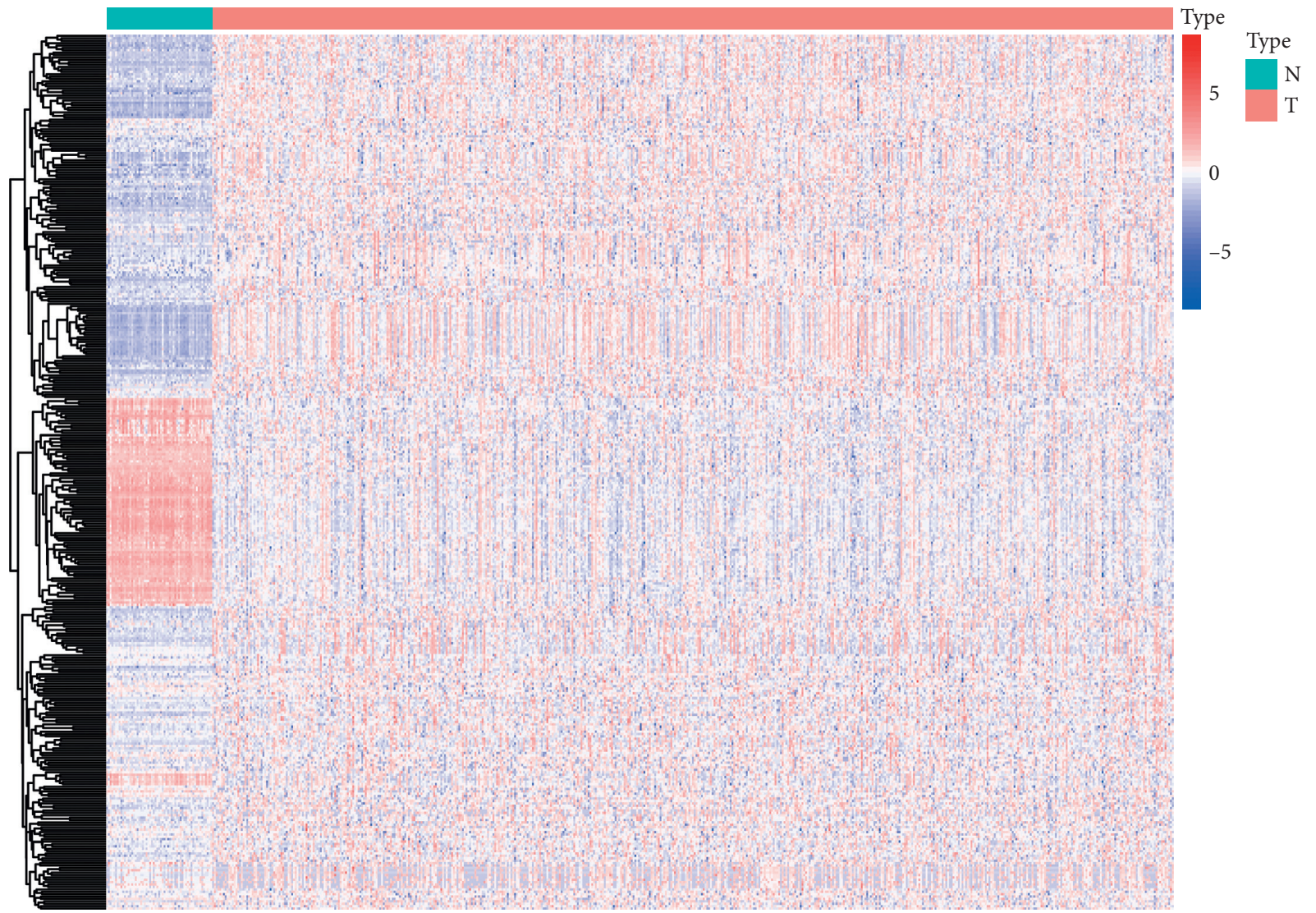

(a)
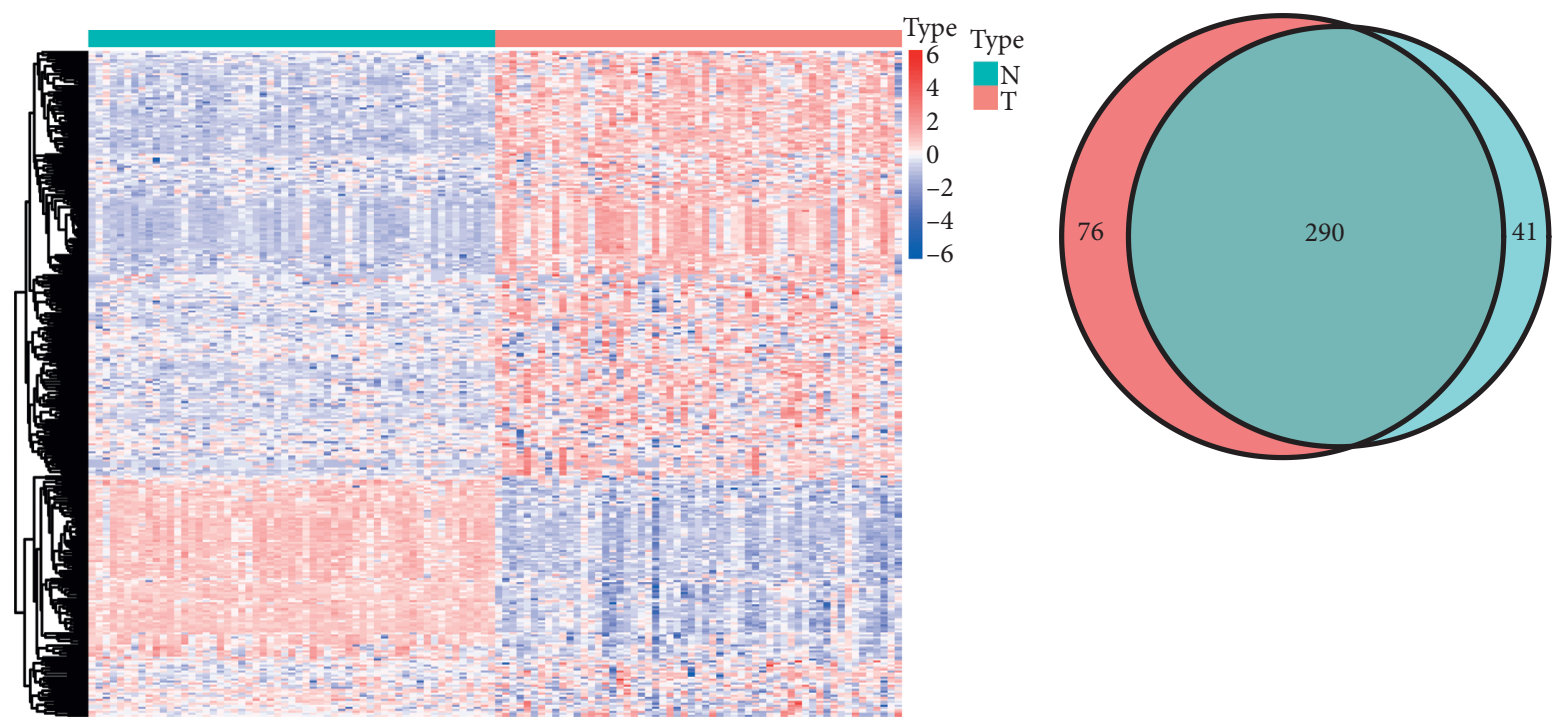

(b)

(c)

Figure 2: Differential expression analyses of redox-associated genes. (a) Heat map of differentially expressed redox-associated genes between overall lung adenocarcinoma tissues and normal lung tissues. (b) Heat map of differentially expressed redox-associated genes between paired lung adenocarcinoma tissues and peritumoral lung tissues. (c) Venn diagram of overlapping redox-associated genes in the two differential expression analyses.

(Figures 7(a) and 7(b)). The low-risk group had a higher rate of mutated EGFR $(15.38 \%$ vs $8.9 \%$, $p$ value $=0.031)$ and a lower rate of mutated TP53 (35.04\% vs $51.69 \%$, $p$ value $<0.001$ ) than the high-risk group (Table 2). Gene set enrichment analysis revealed that the prognostic model was mainly involved in the regulation of the cell cycle, DNA replication and repair, $\mathrm{NADH}$ metabolism, and the p53 signaling pathway (Figures $7(\mathrm{c})$ and $7(\mathrm{~d})$ ). 


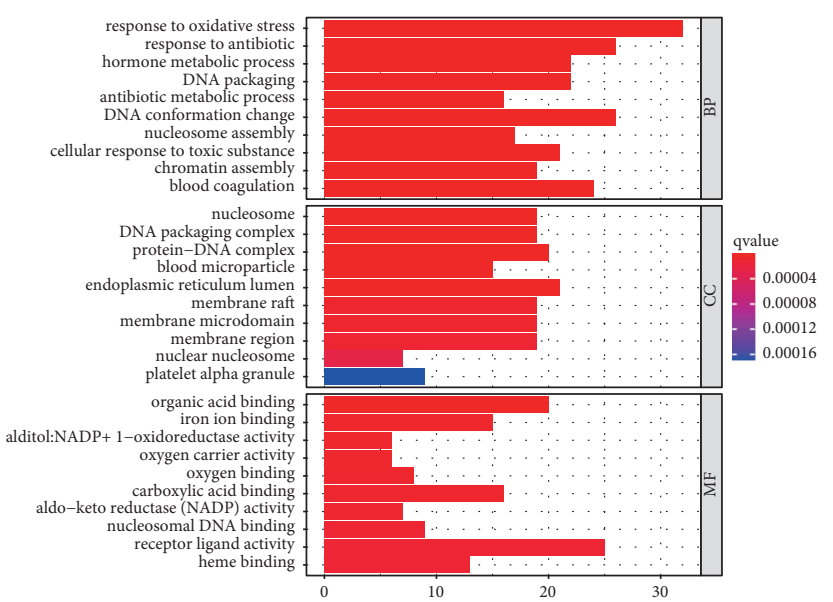

(a)

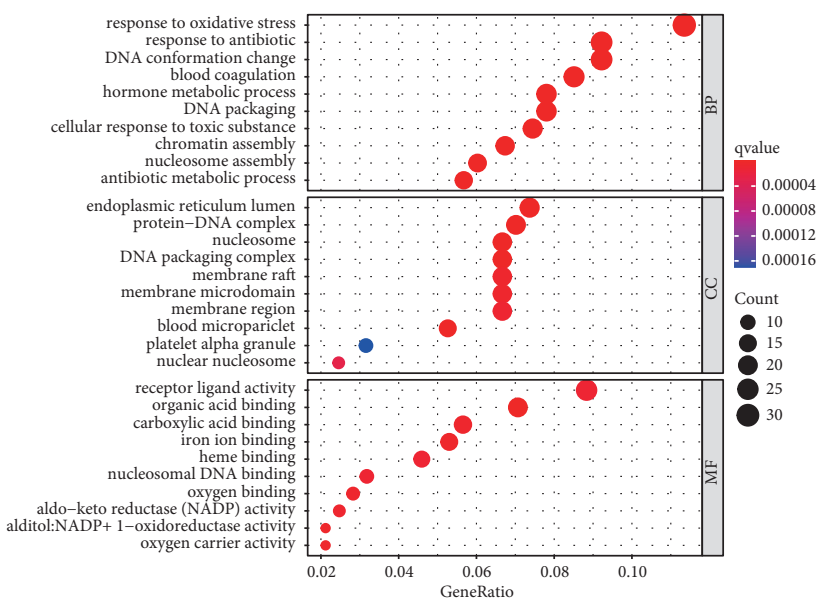

(c)

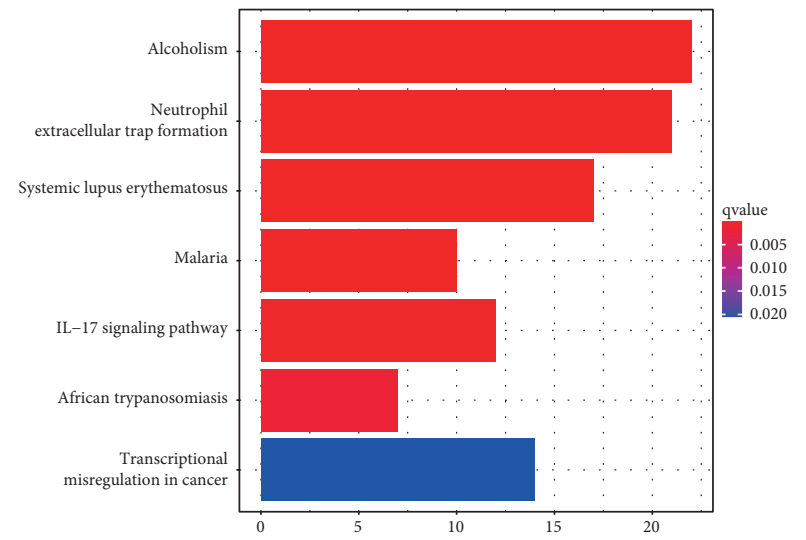

(b)

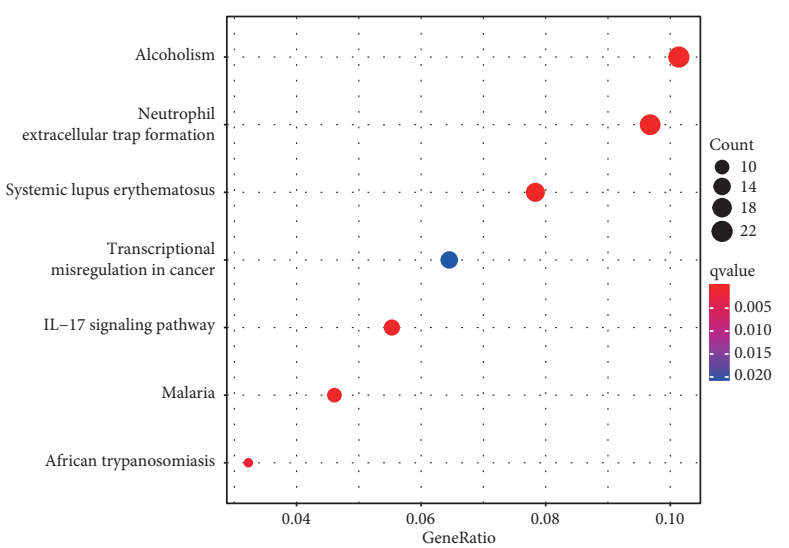

(d)

FIGURE 3: Involved GO terms and pathways of the dysregulated redox-associated genes. (a) Bar plots for the involved GO terms of the dysregulated redox-associated genes. (b) Bar plots for the pathways involved in the dysregulated redox-associated genes. (c) A bubble chart for the involved GO terms of the dysregulated redox-associated genes. (d) A bubble chart for the involved pathways of the dysregulated redox-associated genes. GO=Gene Ontology.

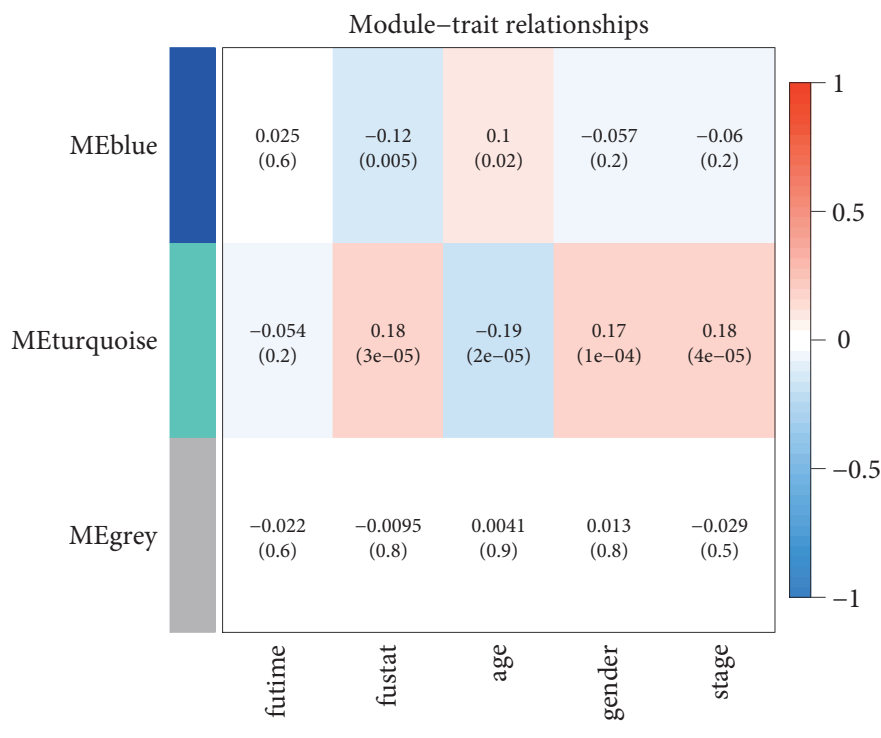

(a)

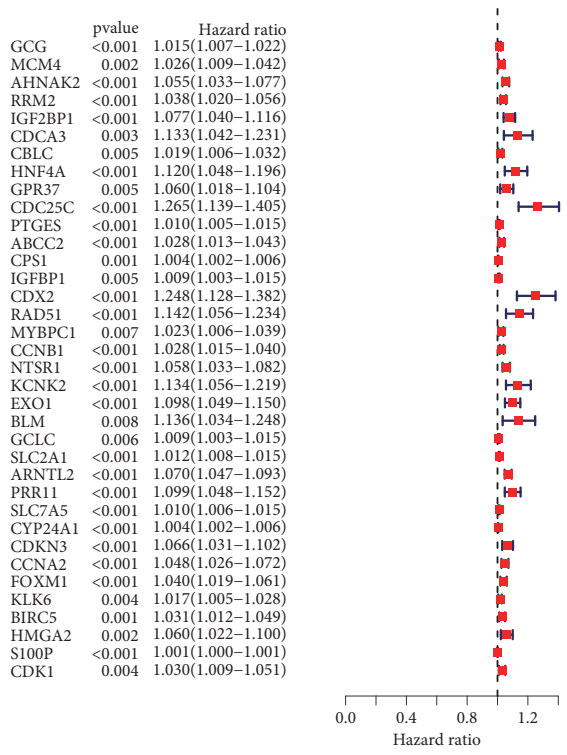

(b)

FIgURE 4: Continued. 


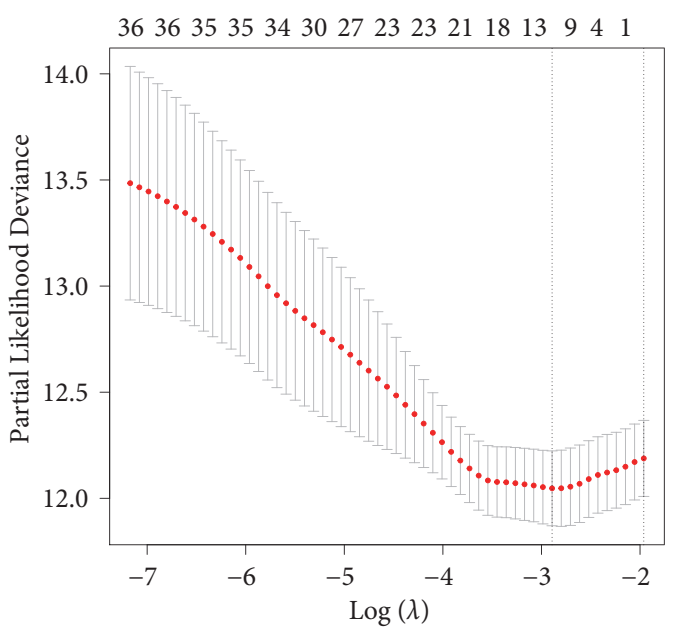

(c)

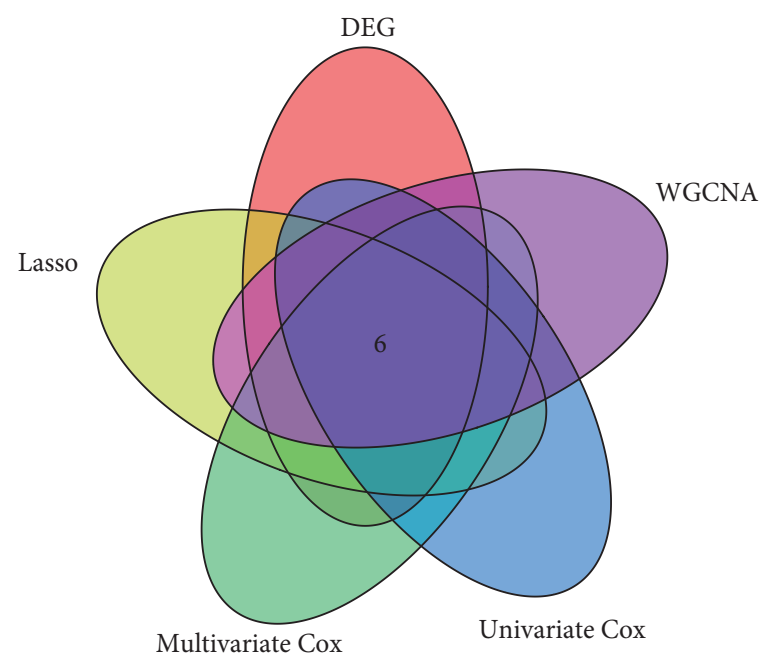

(d)

FIgUre 4: Construction of a prognostic model. (a) Three modules associated with clinical variables in WGCNA. (b) Forest graph of 36 prognostic redox-associated genes obtained by univariate Cox analysis. (c) Ten candidate prognostic genes identified by the LASSO Cox regression analysis. (d) Venn diagram of overlapping prognostic genes in WGCNA, univariate Cox analysis, LASSO Cox regression analysis, and multivariate Cox regression analysis. WGCNA = weighted correlation network analysis; LASSO = shrinkage and selection operator.

TABLE 1: Clinicopathological variables of the discovery cohort and validation cohort.

\begin{tabular}{|c|c|c|}
\hline Characteristics & Discovery cohort $(N=479)$ & Validation cohort $(N=456)$ \\
\hline \multicolumn{3}{|c|}{ Age (years), $n(\%)$} \\
\hline$<65$ & $213(44.47)$ & $210(46.05)$ \\
\hline$\geq 65$ & $266(55.53)$ & $246(53.95)$ \\
\hline \multicolumn{3}{|c|}{ Gender, $n(\%)$} \\
\hline Female & $260(54.28)$ & $208(45.61)$ \\
\hline Male & $219(45.72)$ & $248(54.39)$ \\
\hline \multicolumn{3}{|c|}{ Stage, $n(\%)$} \\
\hline I & $259(54.07)$ & $308(67.54)$ \\
\hline II & $117(24.43)$ & $117(25.66)$ \\
\hline III & $78(16.28)$ & $27(5.92)$ \\
\hline IV & $25(5.22)$ & $4(0.88)$ \\
\hline \multicolumn{3}{|c|}{ Survival status, $n(\%)$} \\
\hline Dead & $177(36.95)$ & $238(52.19)$ \\
\hline Alive & $302(63.05)$ & $218(47.81)$ \\
\hline
\end{tabular}

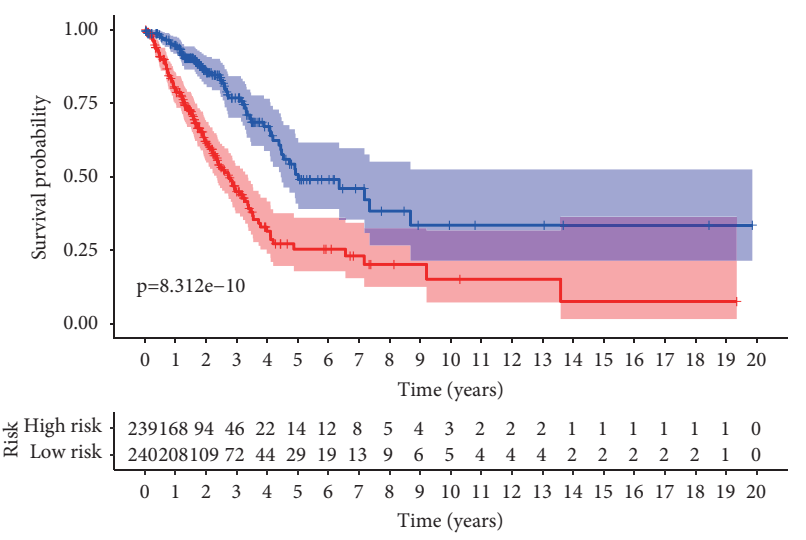

Risk

- High risk

+ Low risk

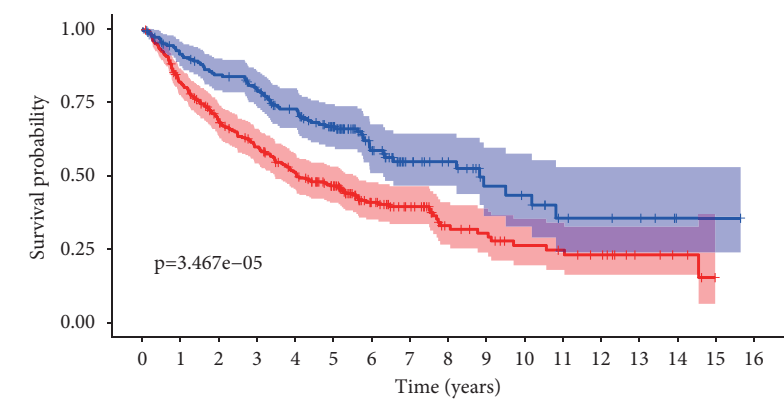

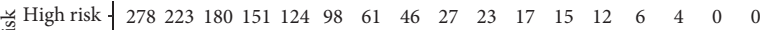
Low risk \begin{tabular}{rrrrrrrrrrrrrrrrrr}
178 & 157 & 143 & 131 & 113 & 90 & 53 & 31 & 25 & 15 & 13 & 7 & 6 & 5 & 1 & 1 & 0 \\
\hline 0 & 1 & 2 & 3 & 4 & 5 & 6 & 7 & 8 & 9 & 10 & 11 & 12 & 13 & 14 & 15 & 16
\end{tabular} Time (years)

Risk 


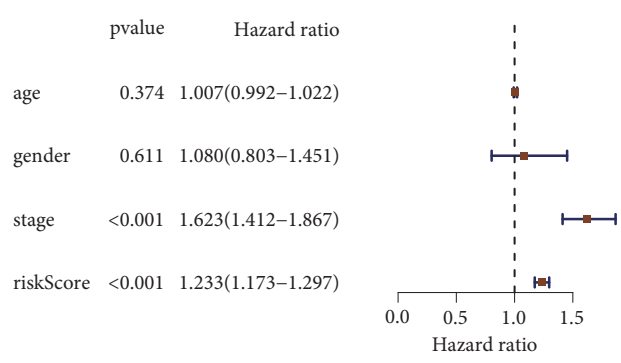

(c)

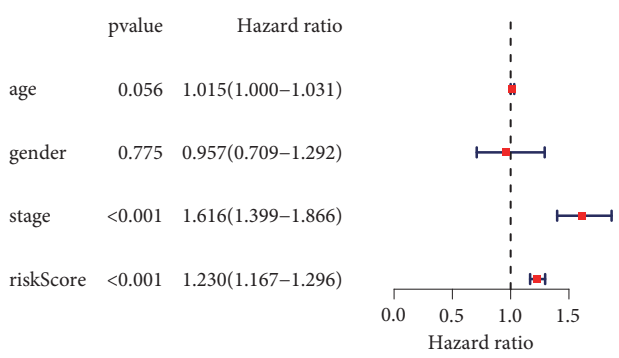

(e)

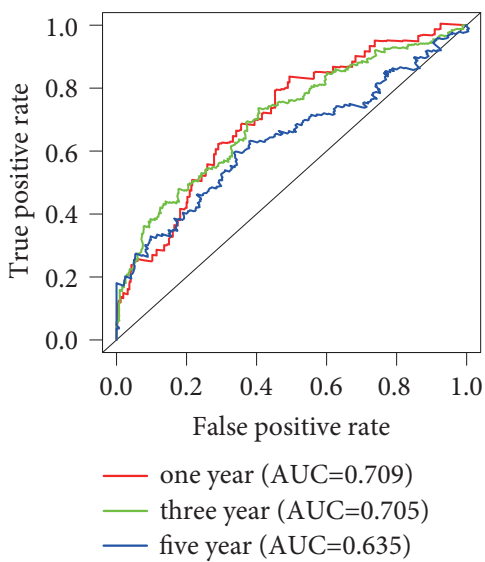

(g)

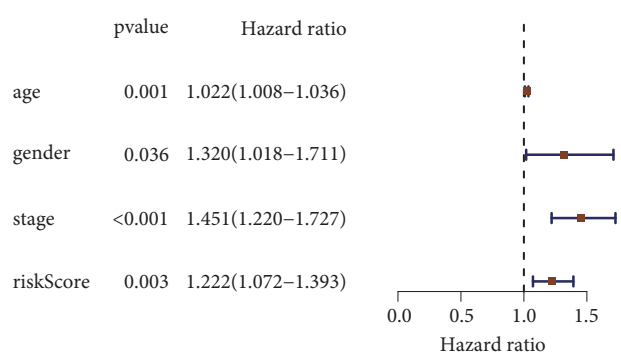

(d)

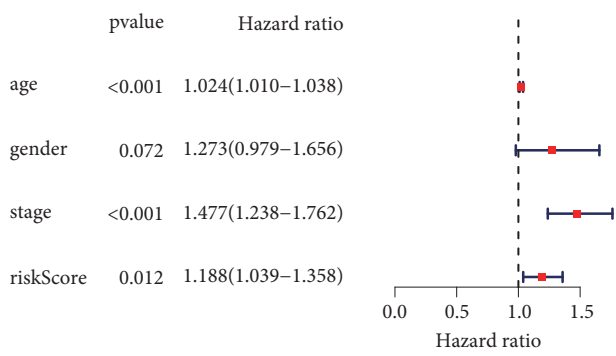

(f)

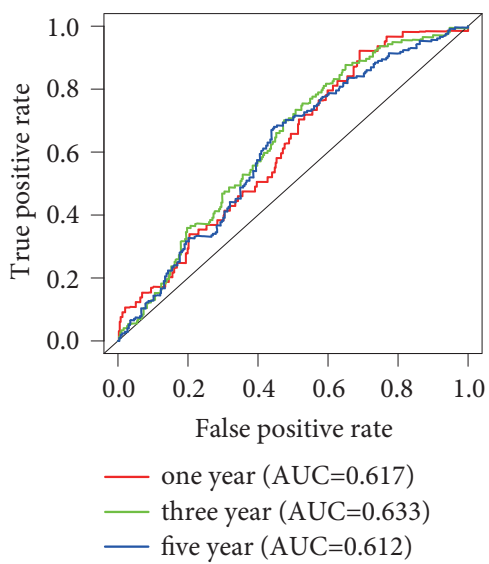

(h)

FIgURE 5: Validation of the prognostic model in the TCGA and GEO cohorts. (a) Survival curves of the low- and high-risk groups in the TCGA cohort. (b) Survival curves of the low- and high-risk groups in the GEO cohort. (c) Univariate Cox analysis of risk score and clinical variables in the TCGA cohort. (d) Univariate Cox analysis of risk score and clinical variables in the GEO cohort. (e) Multivariate Cox analysis of risk score and clinical variables in the TCGA cohort. (f) Multivariate Cox analysis of risk score and clinical variables in the GEO cohort. (g) ROC curves of the prognostic model for predicting 1-, 3-, and 5-year survival rates in the TCGA cohort. (h) ROC curves of the prognostic model for predicting 1-, 3-, and 5-year survival rates in the GEO cohort. TCGA = The Cancer Genome Atlas; GEO=Gene Expression Omnibus.

3.6. The Prognostic Model Was Related to Infiltration of Lymphocytes. We analyzed the infiltration of lymphocytes to gain an insight into the immune implications of the prognostic model. The immune score and stromal score were higher in the low-risk group than that in the high-risk group $(p<0.001)$ (Figures $8(b)$ and $8(c))$. The abundance of immune cells such as B cells, $\mathrm{CD} 4^{+} \mathrm{T}$ cells, dendritic cells, and mast cells was higher in the low-risk group than that in the high-risk group $(p<0.001)$ (Figures $8(\mathrm{a}), 8(\mathrm{~d}), 8(\mathrm{e}), 8(\mathrm{f})$, and $8(\mathrm{~g}))$. Thus, ICIs may achieve better therapeutic effects in the low-risk group since tumor-infiltrating immune cells are essential for the response to ICIs.
3.7. Established Nomogram Could Predict Survival Accurately. Next, we established a nomogram to visualize the prognostic model for clinical application (Figure 9(a)). High prediction accuracy of the nomogram was reflected by calibration curves at 3 and 5 years in the TCGA (Figures 9(b) and 9(c)) and GEO (Figures 9(d) and 9(e)) cohorts.

\section{Discussion}

Patients with LUAD have poor prognosis because of the presence of distant metastases. Only 7\% of LUAD patients with distant metastases can survive 5 years or longer [1]. The carcinogenesis mechanism of LUAD is complex and 


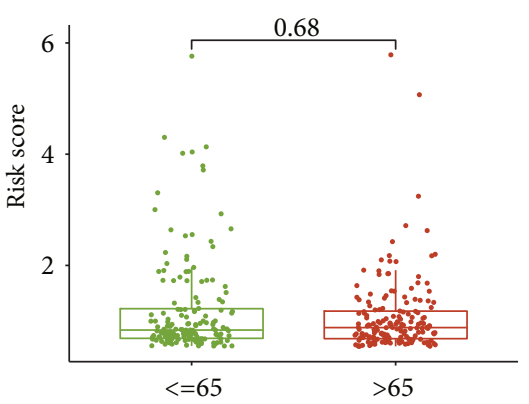

age

age

审 $<=65$

白 $>65$

(a)

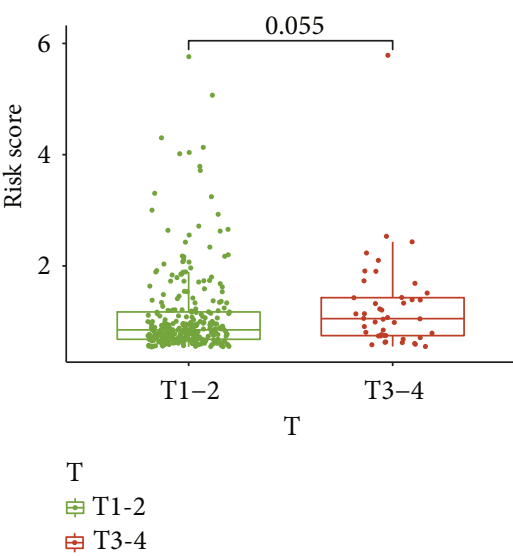

(d)

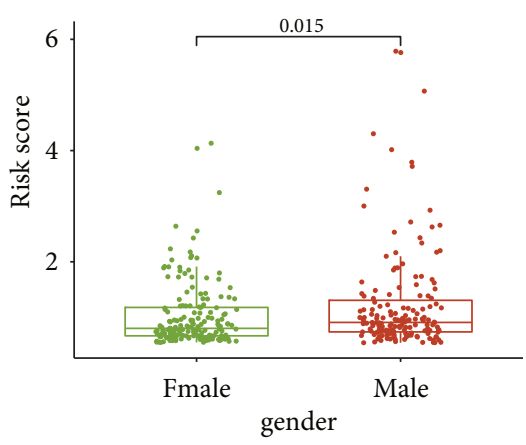

gender

由 Fmale

白 Male

(b)

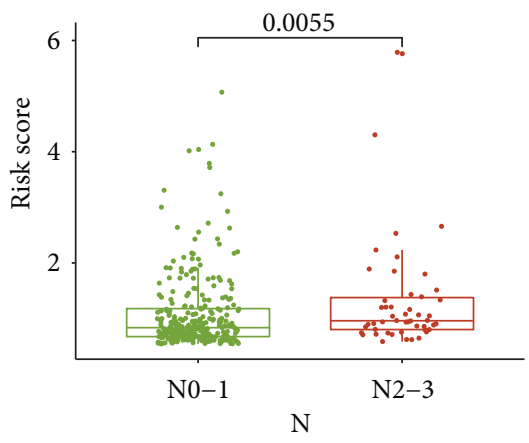

$\mathrm{N}$

白 $\mathrm{N} 0-1$

白 $\mathrm{N} 2-3$

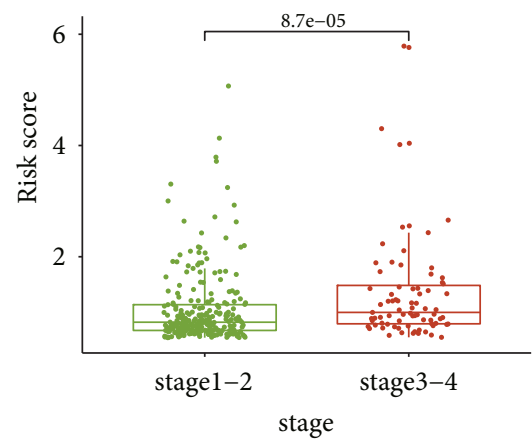

stage

审 stage 1-2

白 stage $3-4$

(c)

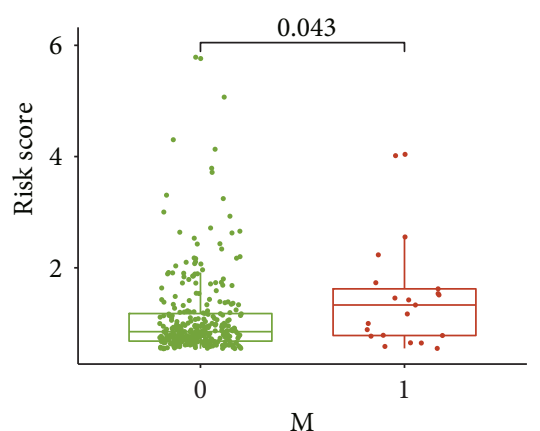

$\mathrm{M}$

由 0

追 1

(e)

(f)

Figure 6: (a-f) Distribution of risk score between patients classified by clinical parameters (age, gender, stage, T stage, $\mathrm{N}$ stage, $\mathrm{M}$ stage).

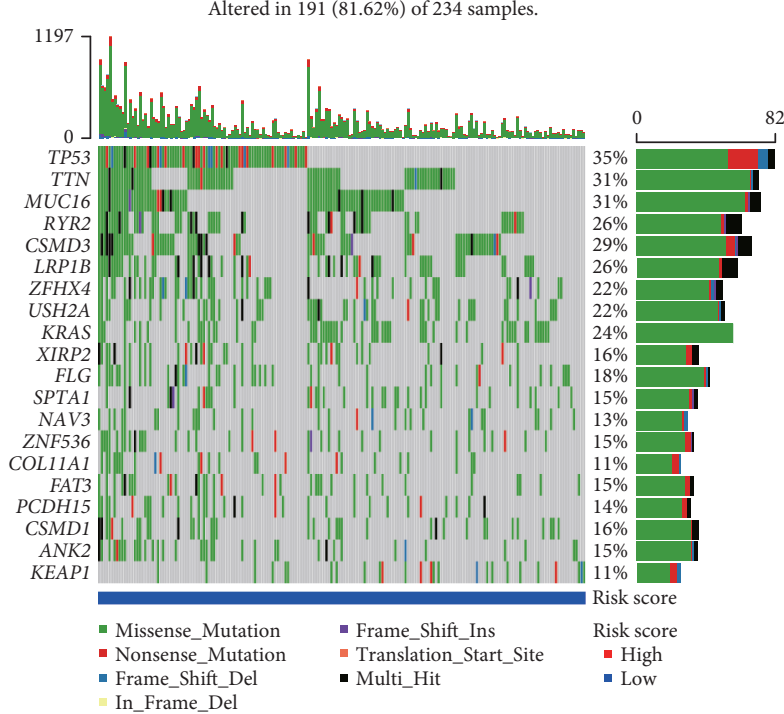

(a)

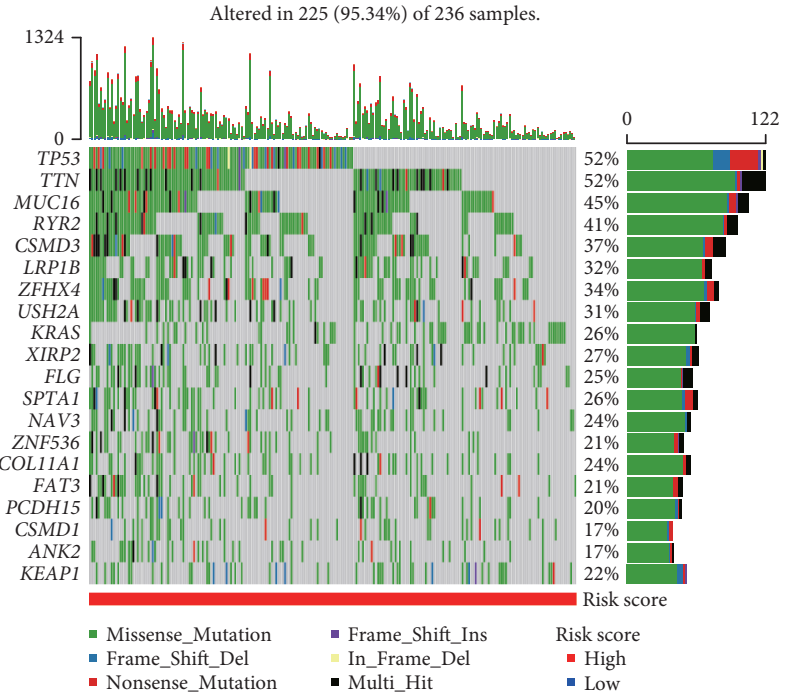

(b)

FIGURE 7: Continued. 

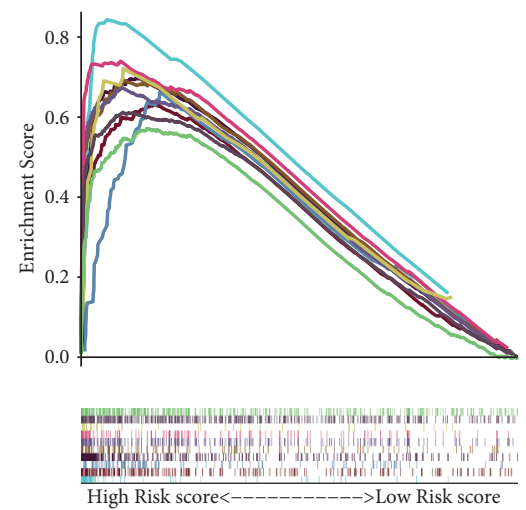

- GOBP_CELL_CYCLE_DNA_REPLICATION

- GOBP_CELL_CYCLE_G2_M_PHASE_TRANSITION

- GOBP_CELLULAR_RESPONSE_TO_HEAT

- GOBP_DNA_REPLICATION

GOBP_MICROTUBULE_CYTOSKELETON

- ORGANIZATION_INVOLVED_IN_MITOSIS

- GOBP_MITOTIC_NUCLEAR_DIVISION

GOBP_MITOTIC_SISTER_CHROMATID

SEGREGATION

- GOBP_NADH_METABOLIC_PROCESS

- GOBP_ORGANELLE_FISSION

_ GOBP_REGULATION_OF_CELL_CYCLE_

PHASE_TRANSITION
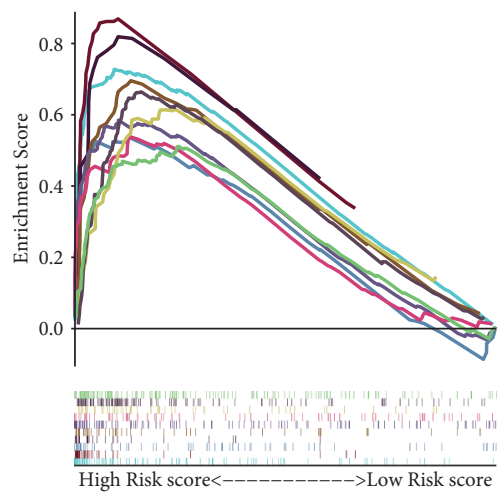

- KEGG_CELL_CYCLE

- KEGG_DNA_REPLICATION

- KEGG_GLYCOLYSIS_GLUCONEOGENESIS

- KEGG_MISMATCH_REPAIR

- KEGG_NUCLEOTIDE_EXCISION_REPAIR

- KEGG_OOCYTE_MEIOSIS

- KEGG_P53_SIGNALING_PATHWAY

- KEGG_RNA_DEGRADATION

- KEGG_SPLICEOSOME

- KEGG_UBIQUITIN_MEDIATED_PROTEOLYSIS

(c)

(d)

Figure 7: (a) Mutation status of genes with a high mutation rate in the low-risk group. (b) Mutation status of genes with a high mutation rate in the high-risk group. (c) Involved GO terms of the prognostic model. (d) Involved pathways of the prognostic model. GO=Gene Ontology.

TABLE 2: Comparison of the mutation rates of TP53 and EGFR between the low- and high-risk groups by the chi-square test.

\begin{tabular}{lccc}
\hline Variables & High risk & Low risk & $\chi^{2}$ \\
\hline \\
Wild & & TP53 & \\
Mutant & $114(48.31 \%)$ & $152(64.96 \%)$ & 13.263 \\
& $122(51.69 \%)$ & $82(35.04 \%)$ & \\
Wild & $215(91.1 \%)$ & EGFR & 4.039 \\
Mutant & $21(8.9 \%)$ & $36(15.38 \%)$ & 0.031 \\
\hline
\end{tabular}

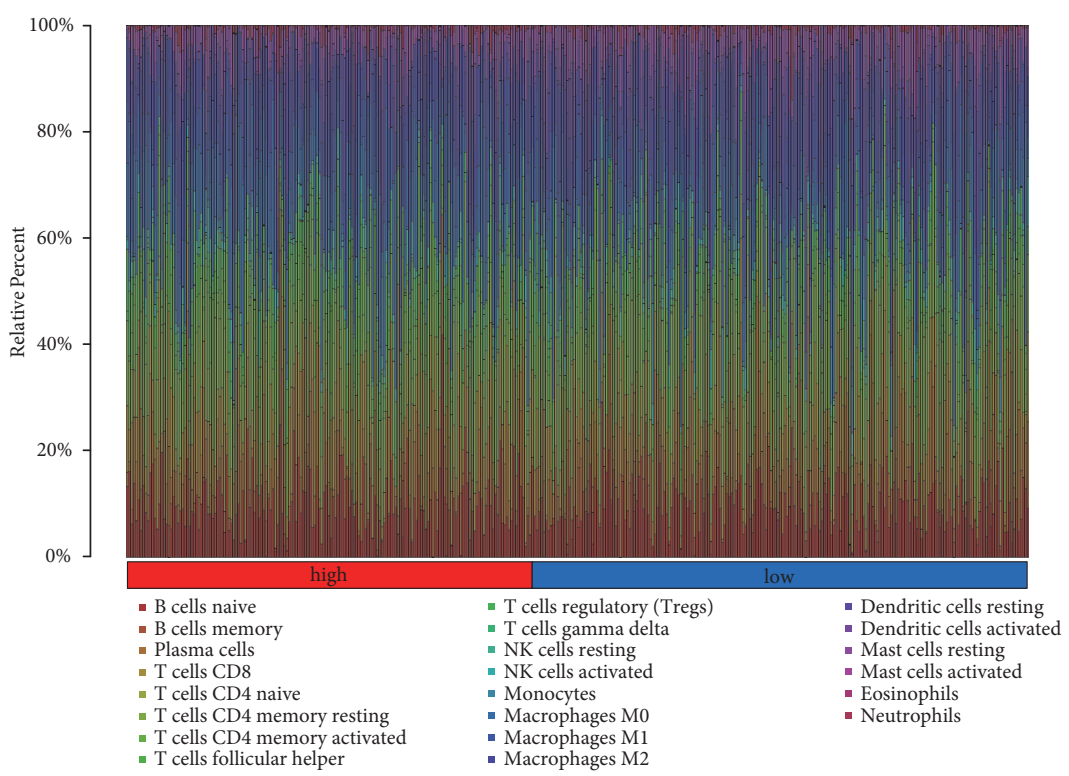

(a)

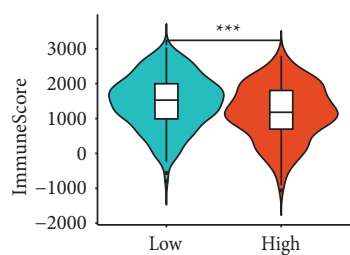

Risk

$\square$ Low

Figure 8: Continued. 


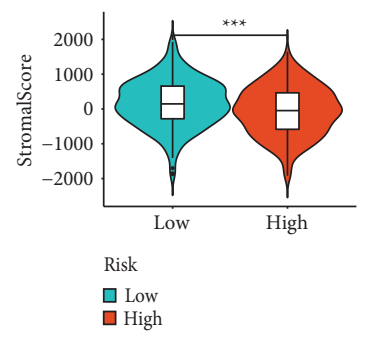

(c)

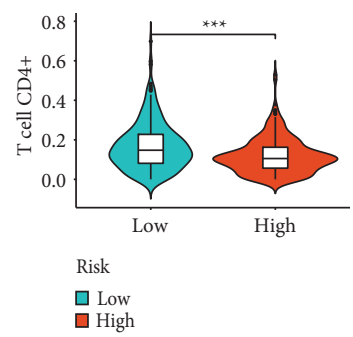

(e)

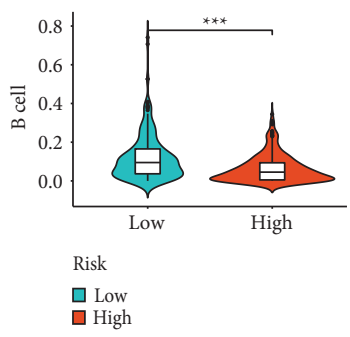

(d)

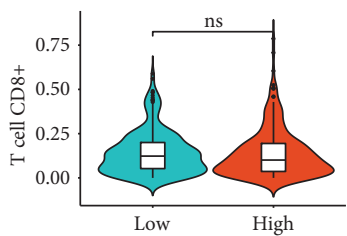

Risk

$\square$ Low
$\square$ High

(f)

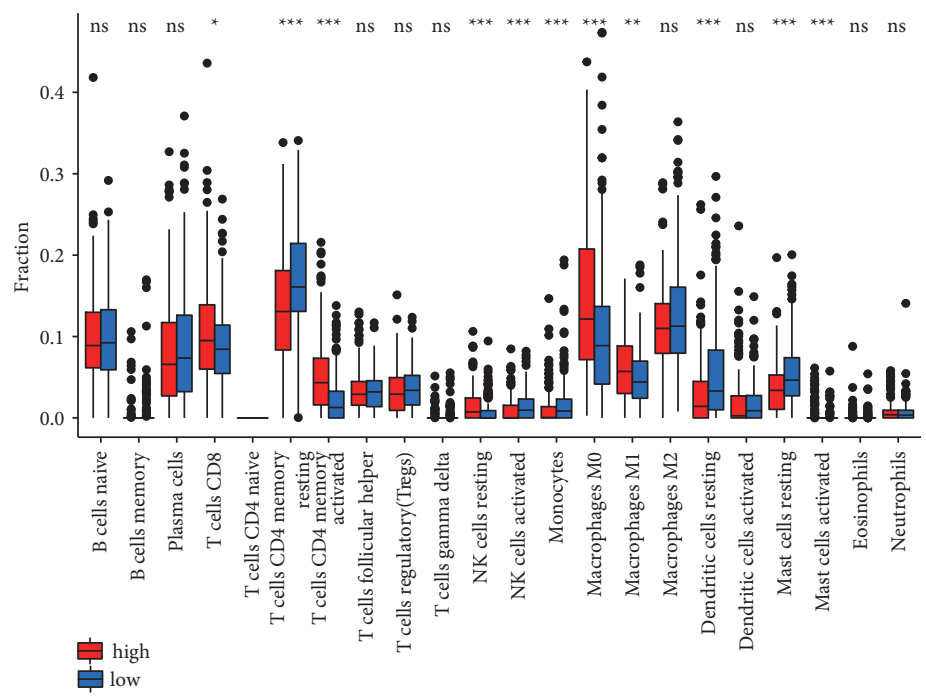

(g)

Figure 8: Correlation of the prognostic model with immunophenotypes. (a) Composition of immune cells of each sample in the low- and high-risk groups. (b-f) Immune score, stromal score, B cells, CD4+ T cells, and CD8+ T cells between the low- and high-risk groups. (g) Proportion of immune cells between the low- and high-risk groups.

involves various biological processes, among which redox is a critical part. Redox can affect the function of proteins or lead to protein degradation by modifying the thiol side chain of protein cysteine residues. Even fine-tuning of redox homeostasis can incur changes in cellular functions such as proliferation, differentiation, and immune response [7, 30, 31]. Redox has been proven to be a significant contributing factor of oncogenesis. However, no study has explored the role of redox-associated genes in lung adenocarcinoma.

In this work, we conducted a systematic analysis to identify novel prognostic biomarkers of LUAD based on datasets in TCGA and GEO. First, 290 dysregulated redoxassociated genes were identified and found to be related to oxidative stress, NADP activity, oxygen binding, DNA packaging, nucleosome assembly, and transcriptional misregulation in cancer. The functions of these genes reflected that redox-associated genes were involved in not only redox actions but also the regulation of DNA replication and transcription. Then, the stepwise analyses identified six prognostic redox-associated genes (AHNAK2, CDC25 C, CPS1, CDX2, NTSR1, and SLC2A1) and constructed a prognostic model. These six redox-associated genes are all oncogenes that could adversely affect survival. Among the six redox-associated genes, NTSR1 did not reach statistical significance in the multivariate Cox regression analysis. The effect of NTSR1 on survival may be dependent on other factors, such as downstream target genes or upstream regulatory genes. Although the expression of NTSR1 is not independently associated with survival, it may represent a 
Points

AHNAK2

CDC25C

CPS1

$\mathrm{CDX} 2$

NTSR1

SLC2A1

Total points

3-year survival

5-year survival $\begin{array}{lllllllllll}0 & 10 & 20 & 30 & 40 & 50 & 60 & 70 & 80 & 90 & 100\end{array}$

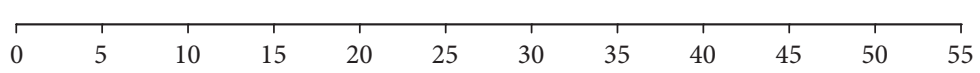

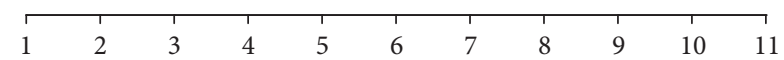

\begin{tabular}{rrrrrrrrrrrr}
\hline 50 & 0 & 50 & 100 & 150 & 200 & 250 & 300 & 350 & 400 & 450 & 500
\end{tabular}

\begin{tabular}{lllllllllllll}
\hline 2 & 3 & 4 & 5 & 6 & 7 & 8 & 9 & 10 & 11 & 12 & 13 & 14
\end{tabular}

$$
\begin{array}{lllllllllllll}
\hline 0 & 5 & 10 & 15 & 20 & 25 & 30 & 35 & 40 & 45 & 50 & 55 & 60
\end{array}
$$

\begin{tabular}{rrrrrrrrrrrrrr}
\hline-20 & 0 & 20 & 40 & 60 & 80 & 100 & 120 & 140 & 160 & 180 & 200 & 220 & 240
\end{tabular}

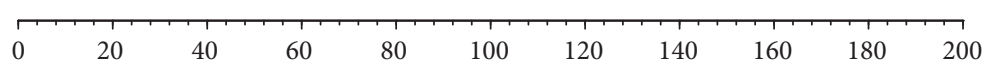

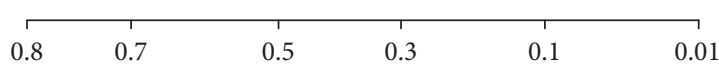

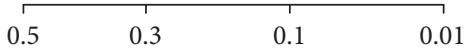

(a)

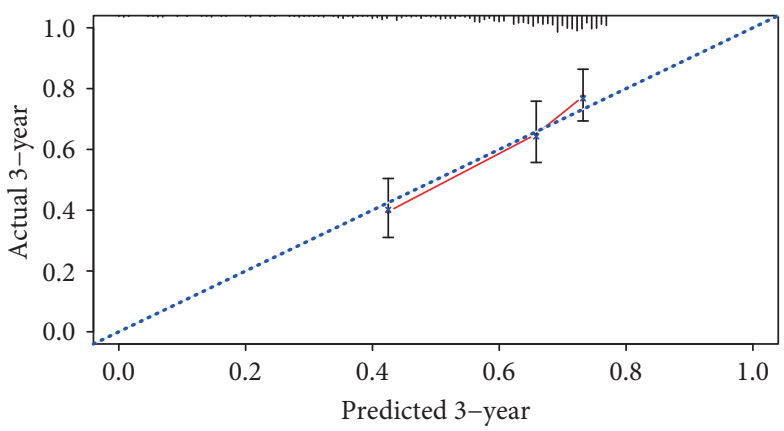

(b)

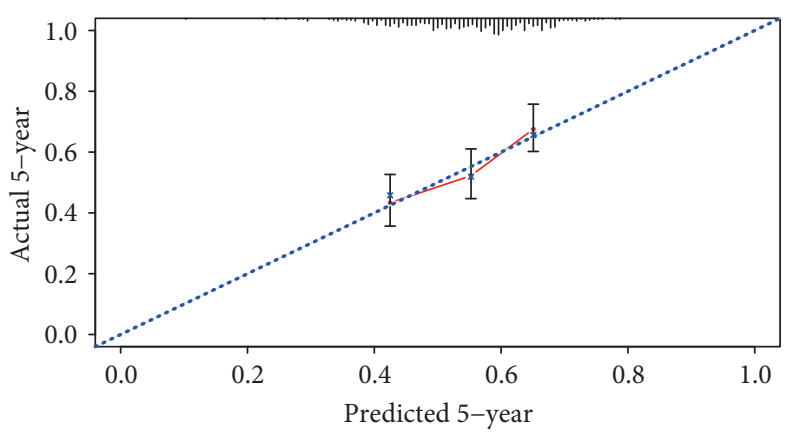

(c)

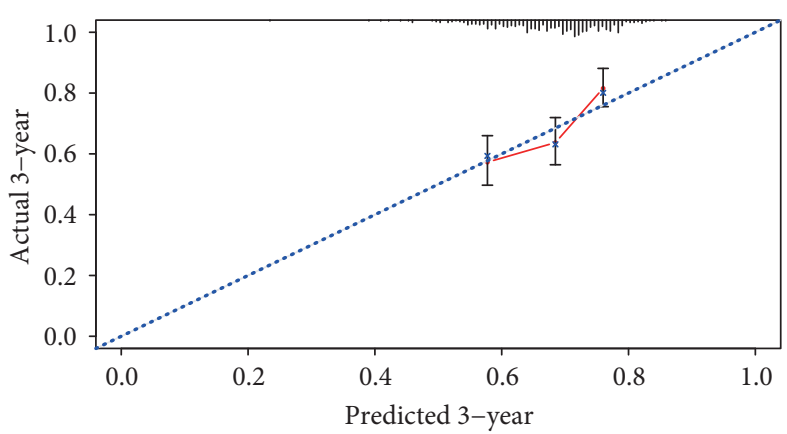

(d)

FIgURE 9: Continued. 


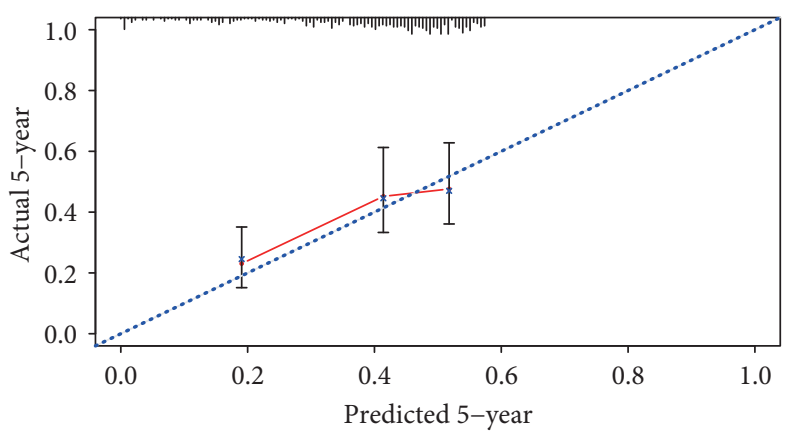

(e)

FIgURE 9: Visualization and verification of the prognostic model. (a) Nomogram based on the prognostic model for survival prediction. (b) Calibration curves showing the accuracy of the nomogram for predicting 3-year survival rates in the TCGA cohort. (c) Calibration curves showing the accuracy of the nomogram for predicting 5-year survival rates in the TCGA cohort. (d) Calibration curves showing the accuracy of the nomogram for predicting 3-year survival rates in the GEO cohort. (e) Calibration curves showing the accuracy of the nomogram for predicting 5-year survival rates in the GEO cohort. TCGA = The Cancer Genome Atlas; GEO= Gene Expression Omnibus.

class of genes with similar effects on survival since it was selected as a significant prognostic gene in both LASSO and multivariate Cox regression analyses.

AHNAK2 belongs to the AHNAK family and was reported to act as an oncogene in papillary thyroid carcinoma, pancreatic ductal adenocarcinoma, and clear cell renal cell carcinoma [32-34]. AHNAK2 was found to facilitate invasion and migration in uveal melanoma and LUAD $[35,36]$. Additionally, AHNAK2 was correlated with immune infiltration in LUAD and papillary thyroid cancer [37, 38]. CDC25C encodes the cell division cycle $25 \mathrm{C}$ protein, serving as an activator of cyclin-dependent kinase 1, which regulates $\mathrm{G} 2 / \mathrm{M}$ cell cycle transition. $\mathrm{CDC} 25 \mathrm{C}$ is essential to the regulation of cell cycle and DNA damage repair. The overexpression of CDC25 C aided in the evasion of cell death in nonmelanoma skin cancer [39]. CDC25 C is upregulated in many tumors and associated with unfavorable outcome [40-44]. CPS1 encodes carbamoyl phosphate synthetase 1, a key enzyme regulating the urea cycle. The inhibition of CPS1 in lung cancer cells carrying KRAS/LKB1 mutations suppressed tumor growth [45]. The downregulation of CPS1 was related to unfavorable survival in hepatocellular carcinoma [46]. Consistent with our findings, CPS1 was identified as an oncogene in LUAD, and the knockdown of CPS1 could suppress cell growth and enhance the efficacy of gemcitabine [47]. Interestingly, evidence has shown that p53 can downregulate CPS1 to inhibit tumor growth [48]. Studies have shown that caudal type homeobox 2 (CDX2) acts as a tumor suppressor gene to inhibit malignant phenotypes of colorectal cancer and that the low expression of CDX2 is correlated with poor survival [49-52]. Wang et al. found that CDX2 was able to activate natural killer cells to enhance the immune response in head and neck squamous cell carcinoma by upregulating CXCL14 [53]. Neurotensin receptor 1 (NTSR1) can combine with neurotensin (NTS) to form a complex to promote tumor progression in solid tumors such as prostate cancer, colorectal cancer, and pancreatic cancer [54-56]. The overexpression of NTSR1 has been shown to indicate poor survival of patients with hepatocellular carcinoma and result in the activation of EGFR [57]. SLC2A1 encodes GLUT-1 protein, which is an important glucose transporter in glucose metabolism. Evidence suggests that GLUT-1 is upregulated in prostate cancer and that the knockdown of GLUT-1 leads to proliferation inhibition and cell cycle arrest of prostate cancer cells [58]. SLC2A1 was identified as an adverse prognostic factor of gastric cancer [59]. A recent study also demonstrated that $S L C 2 A 1$ was overexpressed in LUAD and was associated with shorter survival of patients, which was consistent with our findings $[60,61]$.

Then, a series of analyses were conducted to verify the prognostic performance of the model. First, the differential expression of the prognostic genes in the model was validated in GSM43458 and GSM32863. Then, the prognostic value of these genes in the model was validated in GEPIA, and the results confirmed that all of the genes were oncogenes adversely affecting survival. Next, the robust prognostic performance of the model was demonstrated in the TCGA and GEO cohorts (GSE37745, GSE31210, and GSE50081). The results of the Kaplan-Meier survival curves and the univariate Cox regression analysis in both the TCGA and GEO cohorts showed that patients with low-risk score had a better prognosis. The multivariate Cox analysis further indicated that the risk score based on our model was an independent risk factor for survival. The AUCs for 1, 3, and 5 years in the TCGA and GEO cohorts suggested that the prognostic model possesses robust predictive power.

We also evaluated the relationship of the prognostic model with clinicopathological variables. The low-risk score was associated with early stage, low mutation rate of TP53, and high mutation rate of EGFR, which supported our findings that the patients with low-risk score had favorable survival. TP53 encodes p53, which is a common tumor suppressor protein. The mutation of TP53 can lead to loss of its function in tumor suppression and was proven to be correlated with unfavorable prognosis in lung cancer $[62,63]$. A clinical trial suggested that the mutation of TP53 indicated poor survival in lung cancer patients receiving chemotherapy after surgery [64]. Patients with mutations in EGFR are more likely to respond to tyrosine kinase 
inhibitors. GSEA revealed that the prognostic model was mainly involved in the regulation of cell cycle, DNA replication and repair, NADH metabolism, and p53 signaling pathway, which are vital processes affecting the proliferation of cancer cells and tumor progression.

Since redox can affect the function of lymphocytes and immune response $[7,65]$, we speculated that the prognostic model was associated with antitumor immunity. We evaluated the correlation between the prognostic model and immunophenotypes to find out that the low-risk score was associated with high infiltration of lymphocytes such as $\mathrm{B}$ cells, $\mathrm{CD} 4^{+} \mathrm{T}$ cells, and dendritic cells. The abundance of tumor-infiltrating lymphocytes was positively related to the efficacy of immune checkpoint inhibitors [66, 67]. B cells produce immunoglobulins and regulate $\mathrm{T}$ cells to enhance the antitumor response [68]. $\mathrm{CD}^{+} \mathrm{T}$ cells can increase immune response by activating $\mathrm{CD}^{+} \mathrm{T}$ cells $[69,70]$. The antigen-presenting function of dendritic cells is essential for the activation of T cells. These results suggested that patients with low-risk score may have stronger antitumor immunity, and immunotherapy may work better in these patients than in those with high-risk score. Our model can be a potential tool to identify proper candidates for immunotherapy.

Limitations of our study are listed as follows. First, since our study was a retrospective study based on the analyses of data in public databases, the prognostic performance of the model constructed in our study needs to be tested in a prospective cohort study. Second, mechanistic exploration was not performed in our study, which needs to be conducted by in vivo and in vitro studies to explain the exact role of prognostic redox-associated genes in tumorigenesis and tumor immunity.

\section{Conclusions}

In conclusion, we introduced a novel redox-based prognostic model, which had robust prognostic performance in LUAD. The prognostic model can be a potential theranostic indicator that aids in predicting the survival of patients and identifying proper candidates for immunotherapy. Our findings explored novel prognostic biomarkers and contributed to tailoring optimal treatment strategies for patients with LUAD.

\section{Abbreviations}

$\begin{array}{ll}\text { abrLUAD: } & \text { lung adenocarcinoma } \\ \text { TCGA: } & \text { The Cancer Genome Atlas } \\ \text { WGCNA: } & \begin{array}{l}\text { weighted correlation network analysis } \\ \text { least absolute shrinkage and selection } \\ \text { operator }\end{array} \\ \text { RASSO: } & \begin{array}{l}\text { receiver operating characteristic curve } \\ \text { gene set enrichment analysis }\end{array} \\ \text { GSEA: } & \text { immune checkpoint inhibitor } \\ \text { ICI: } & \text { reactive oxygen species } \\ \text { ROS: } & \text { NADPH oxidases } \\ \text { NOXs: } & \text { Gene Expression Omnibus } \\ \text { GEO: } & \text { false discovery rate } \\ \text { FDR: } & \text { Gene Ontology } \\ \text { GO: } & \end{array}$

KEGG: $\quad$ Kyoto Encyclopedia of Genes and Genomes

GEPIA: Gene Expression Profiling Interactive Analysis

ESTIMATE: Estimation of STromal and Immune cells in Malignant Tumours using Expression data

CIBERSORT: Cell-type Identification By Estimating Relative Subsets Of RNA Transcripts

OS: $\quad$ overall survival

DFS: disease-free survival

HR: hazard ratio

CI: confidence interval

\section{Data Availability}

The datasets analyzed in this study are available at TCGA (https://portal.gdc.cancer.gov/) and GEO (https://www. ncbi.nlm.nih.gov/geo).

\section{Ethical Approval}

Not applicable.

\section{Disclosure}

Lingyan Xiao and Qian Li are co-first authors.

\section{Conflicts of Interest}

The authors declare that there are no conflicts of interest regarding the publication of this study.

\section{Authors' Contributions}

Lingyan Xiao and Bo Liu designed methodology; Lingyan Xiao, Qian Li, and Yongbiao Huang analyzed the data and plotted the figure; Lingyan Xiao wrote the original draft; Yongbiao Huang, Li Ma, and Zhijie Fan wrote, reviewed, and edited the manuscript; Xianglin Yuan and Bo Liu supervised the study; and Bo Liu and Xianglin Yuan have contributed equally to this work. Lingyan Xiao and Qian Li have contributed equally to this work.

\section{Acknowledgments}

This work was supported by grants from the National Natural Science Foundation of China (Grant Nos. 82130092 and 81902619) and the National Natural Science Foundation of Hubei Province (2020CFB591).

\section{Supplementary Materials}

Supplementary Figure 1. Validation of 6 redox-associated genes in GEO. (a) Differential expression of 6 prognostic redox-associated genes in GSE32863. (b) Differential expression of 6 prognostic redox-associated genes in GSE43458. GEO = Gene Expression Omnibus. Supplementary Figure 2. Validation of 6 redox-associated genes in GEPIA. (A-F) Survival curves showing overall survival of patients with LUAD divided by expression of 6 redox-associated genes (AHNAK2, CDC25 C, CPS1, CDX2, NTSR1, 
and SLC2A1). (G-L) Survival curves showing the diseasefree survival of patients with LUAD divided by expression of 6 redox-associated genes (AHNAK2, CDC25 C, CPS1, CDX2, NTSR1, and SLC2A1). GEPIA = Gene Expression Profiling Interactive Analysis. Supplementary Table 1: multivariate Cox regression analysis of prognostic redox-associated genes. Supplementary Table 2: clinical features of the discovery cohort. (Supplementary Materials)

\section{References}

[1] American Cancer Society, 2016, https://www.cancer.org/ cancer/lung-cancer/detection-diagnosis-staging/survivalrates.html.

[2] H. Kong and N. S. Chandel, "Regulation of redox balance in cancer and T cells," Journal of Biological Chemistry, vol. 293, no. 20, pp. 7499-7507, 2018.

[3] A. Ceriello and E. Motz, "Is oxidative stress the pathogenic mechanism underlying insulin resistance, diabetes, and cardiovascular disease? the common soil hypothesis revisited," Arteriosclerosis, Thrombosis, and Vascular Biology, vol. 24, no. 5, pp. 816-823, 2004.

[4] G. Cosentino, I. Plantamura, A. Cataldo, and M. V. Iorio, "MicroRNA and oxidative stress interplay in the context of breast cancer pathogenesis," International Journal of Molecular Sciences, vol. 20, no. 20, Article ID 5143, 2019.

[5] M. Brownlee, "Biochemistry and molecular cell biology of diabetic complications," Nature, vol. 414, no. 6865, pp. 813-820, 2001.

[6] D. P. Preci, A. Almeida, A. L. Weiler, M. L. Mukai Franciosi, and A. M. Cardoso, "Oxidative damage and antioxidants in cervical cancer," International Journal of Gynecological Cancer, vol. 31, no. 2, pp. 265-271, 2021.

[7] J. Muri and M. Kopf, "Redox regulation of immunometabolism," Nature Reviews Immunology, vol. 21, no. 6, pp. 363-381, 2021.

[8] A. Cruz-Gregorio, J. Manzo-Merino, and M. Lizano, "Cellular redox, cancer and human papillomavirus," Virus Research, vol. 246 , pp. $35-45,2018$.

[9] S. Emanuele, A. D’Anneo, G. Calvaruso, C. Cernigliaro, M. Giuliano, and M. Lauricella, "The double-edged sword profile of redox signaling: oxidative events as molecular switches in the balance between cell physiology and cancer," Chemical Research in Toxicology, vol. 31, no. 4, pp. 201-210, 2018.

[10] I. I. C. Chio, S. M. Jafarnejad, M. Ponz-Sarvise et al., "NRF2 promotes tumor maintenance by modulating mRNA translation in pancreatic cancer," Cell, vol. 166, no. 4, pp. 963-976, 2016.

[11] Z. T. Schafer, A. R. Grassian, L. Song et al., "Antioxidant and oncogene rescue of metabolic defects caused by loss of matrix attachment," Nature, vol. 461, no. 7260, pp. 109-113, 2009.

[12] T. Takata, S. Araki, Y. Tsuchiya, and Y. Watanabe, "Oxidative stress orchestrates MAPK and nitric-oxide synthase signal," International Journal of Molecular Sciences, vol. 21, no. 22, p. 8750, 2020.

[13] D. Seth and J. Rudolph, "Redox regulation of MAP kinase phosphatase 3," Biochemistry, vol. 45, no. 28, pp. 8476-8487, 2006.

[14] J. Muri, H. Thut, Q. Feng, and M. Kopf, "Thioredoxin-1 distinctly promotes NF- $\kappa \mathrm{B}$ target DNA binding and NLRP3 inflammasome activation independently of Txnip," Elife, vol. 9, 2020.
[15] N. Koundouros and G. Poulogiannis, "Phosphoinositide 3kinase/akt signaling and redox metabolism in cancer," Frontiers in Oncology, vol. 8, p. 160, 2018.

[16] J. K. M. Lim and G. Leprivier, "The impact of oncogenic RAS on redox balance and implications for cancer development," Cell Death \& Disease, vol. 10, no. 12, p. 955, 2019.

[17] J. Mitsushita, J. D. Lambeth, and T. Kamata, "The superoxidegenerating oxidase Nox1 is functionally required for Ras oncogene transformation," Cancer Research, vol. 64, no. 10, pp. 3580-3585, 2004.

[18] M.-T. Park, M.-J. Kim, Y. Suh et al., "Novel signaling axis for ROS generation during K-Ras-induced cellular transformation," Cell Death \& Differentiation, vol. 21, no. 8, pp. 1185-1197, 2014.

[19] S.-R. Lee, K.-S. Yang, J. Kwon, C. Lee, W. Jeong, and S. G. Rhee, "Reversible inactivation of the tumor suppressor PTEN by H2O2," Journal of Biological Chemistry, vol. 277, no. 23, pp. 20336-20342, 2002.

[20] Y. Zhang, J. Park, S.-J. Han et al., "Redox regulation of tumor suppressor PTEN in cell signaling," Redox Biology, vol. 34, Article ID 101553, 2020.

[21] B. W. L. Lee, P. Ghode, and D. S. T. Ong, "Redox regulation of cell state and fate," Redox Biology, vol. 25, Article ID 101056, 2019.

[22] P. Langfelder and S. Horvath, "WGCNA: an R package for weighted correlation network analysis," BMC Bioinformatics, vol. 9, no. 1, p. 559, 2008.

[23] J. Long, S. Huang, Y. Bai et al., "Transcriptional landscape of cholangiocarcinoma revealed by weighted gene coexpression network analysis," Briefings in Bioinformatics, vol. 22, no. 4, 2021.

[24] J. Wang, S. Cong, H. Wu et al., "Identification and analysis of potential autophagy-related biomarkers in endometriosis by WGCNA," Frontiers in molecular biosciences, vol. 8, Article ID 743012, 2021.

[25] Y. Zhao, T. Ma, and D. Zou, "Identification of unique transcriptomic signatures and hub genes through RNA sequencing and integrated WGCNA and PPI network analysis in nonerosive reflux disease," Journal of Inflammation Research, vol. 14, pp. 6143-6156, 2021.

[26] K. Yoshihara, M. Shahmoradgoli, E. Martínez et al., "Inferring tumour purity and stromal and immune cell admixture from expression data," Nature Communications, vol. 4, no. 1, p. 2612, 2013.

[27] T. Li, J. Fu, Z. Zeng et al., "TIMER2.0 for analysis of tumorinfiltrating immune cells," Nucleic Acids Research, vol. 48, no. W1, pp. W509-w514, 2020.

[28] B. Li, E. Severson, J.-C. Pignon et al., "Comprehensive analyses of tumor immunity: implications for cancer immunotherapy," Genome Biology, vol. 17, no. 1, p. 174, 2016.

[29] A. M. Newman, C. L. Liu, M. R. Green et al., "Robust enumeration of cell subsets from tissue expression profiles," Nature Methods, vol. 12, no. 5, pp. 453-457, 2015.

[30] T. Finkel and N. J. Holbrook, "Oxidants, oxidative stress and the biology of ageing," Nature, vol. 408, no. 6809, pp. 239-247, 2000.

[31] L. Zhang, X. Wang, R. Cueto et al., "Biochemical basis and metabolic interplay of redox regulation," Redox Biology, vol. 26, Article ID 101284, 2019.

[32] Z. Xie, Y. Lun, X. Li et al., "Bioinformatics analysis of the clinical value and potential mechanisms of AHNAK2 in papillary thyroid carcinoma," Aging, vol. 12, no. 18, pp. 18163-18180, 2020. 
[33] Z.-Q. Yang, Y.-J. Liu, and X.-L. Zhou, "An integrated microarray analysis reveals significant diagnostic and prognostic biomarkers in pancreatic cancer," Medical Science Monitor, vol. 26, Article ID e921769, 2020.

[34] M. Wang, X. Li, J. Zhang et al., "AHNAK2 is a novel prognostic marker and oncogenic protein for clear cell renal cell carcinoma," Theranostics, vol. 7, no. 5, pp. 1100-1113, 2017.

[35] G. Liu, Z. Guo, Q. Zhang, Z. Liu, and D. Zhu, "AHNAK2 promotes migration, invasion, and epithelial-mesenchymal transition in lung adenocarcinoma cells via the TGF- $\beta / \mathrm{smad} 3$ pathway," OncoTargets and Therapy, vol. 13, pp. 12893-12903, 2020.

[36] M. Li, Y. Liu, Y. Meng, and Y. Zhu, “AHNAK nucleoprotein 2 performs a promoting role in the proliferation and migration of uveal melanoma cells," Cancer Biotherapy and Radiopharmaceuticals, vol. 34, no. 10, pp. 626-633, 2019.

[37] L. Zheng, S. Li, X. Zheng, R. Guo, and W. Qu, "AHNAK2 is a novel prognostic marker and correlates with immune infiltration in papillary thyroid cancer: evidence from integrated analysis," International Immunopharmacology, vol. 90, Article ID 107185, 2021.

[38] M. Zheng, J. Liu, T. Bian et al., "Correlation between prognostic indicator AHNAK2 and immune infiltrates in lung adenocarcinoma," International Immunopharmacology, vol. 90, Article ID 107134, 2021.

[39] J. Al-Matouq, T. R. Holmes, and L. A. Hansen, "CDC25B and CDC25C overexpression in nonmelanoma skin cancer suppresses cell death," Molecular Carcinogenesis, vol. 58, no. 9, pp. 1691-1700, 2019.

[40] R. Xun, H. Lu, and X. Wang, "Identification of CDC25C as a potential biomarker in hepatocellular carcinoma using bioinformatics analysis," Technology in Cancer Research and Treatment, vol. 19, Article ID 153303382096747, 2020.

[41] Z. Xia, W. Ou-Yang, T. Hu, and K. Du, "Prognostic significance of CDC25C in lung adenocarcinoma: an analysis of TCGA data," Cancer genetics, vol. 233-234, pp. 67-74, 2019.

[42] C. Wu, J. Lyu, E. J. Yang, Y. Liu, B. Zhang, and J. S. Shim, "Targeting AURKA-CDC25C axis to induce synthetic lethality in ARID1A-deficient colorectal cancer cells," Nature Communications, vol. 9, no. 1, p. 3212, 2018.

[43] J. Wei, Y. Yin, Q. Deng et al., "Integrative analysis of MicroRNA and gene interactions for revealing candidate signatures in prostate cancer," Frontiers in Genetics, vol. 11, p. 176, 2020.

[44] X.-q. Wang, S.-w. Xu, W. Wang et al., "Identification and validation of a novel DNA damage and DNA repair related genes based signature for colon cancer prognosis," Frontiers in Genetics, vol. 12, Article ID 635863, 2021.

[45] J. Kim, Z. Hu, L. Cai et al., "CPS1 maintains pyrimidine pools and DNA synthesis in KRAS/LKB1-mutant lung cancer cells," Nature, vol. 546, no. 7656, pp. 168-172, 2017.

[46] D. A. Ridder, M. Schindeldecker, A. Weinmann et al., "Key enzymes in pyrimidine synthesis, CAD and CPS1, predict prognosis in hepatocellular carcinoma," Cancers, vol. 13, no. 4, p. 744, 2021.

[47] M. Çeliktaş, I. Tanaka, S. Chandra Tripathi et al., "Role of CPS1 in cell growth, metabolism, and prognosis in LKB1inactivated lung adenocarcinoma," Journal of the National Cancer Institute, vol. 109, no. 3, pp. djw231-9, 2017.

[48] L. Li, Y. Mao, L. Zhao et al., "p53 regulation of ammonia metabolism through urea cycle controls polyamine biosynthesis," Nature, vol. 567, no. 7747, pp. 253-256, 2019.

[49] J. Zheng, S. He, J. Qi et al., "Targeted CDX2 expression inhibits aggressive phenotypes of colon cancer cells in vitro and in vivo," International Journal of Oncology, vol. 51, no. 2, pp. 478-488, 2017.

[50] J. Yu, D. Liu, X. Sun et al., "CDX2 inhibits the proliferation and tumor formation of colon cancer cells by suppressing $\mathrm{Wnt} / \beta$-catenin signaling via transactivation of GSK-3 $\beta$ and Axin2 expression," Cell Death \& Disease, vol. 10, no. 1, p. 26, 2019.

[51] J. Yu, S. Li, Z. Xu et al., "CDX2 inhibits epithelial-mesenchymal transition in colorectal cancer by modulation of Snail expression and $\beta$-catenin stabilisation via transactivation of PTEN expression," British Journal of Cancer, vol. 124, no. 1, pp. 270-280, 2021.

[52] S. H. den Uil, M. de Wit, R. J. C. Slebos et al., "Quantitative analysis of CDX2 protein expression improves its clinical utility as a prognostic biomarker in stage II and III colon cancer," European Journal of Cancer, vol. 144, pp. 91-100, 2021.

[53] H. Wang, S. Nan, Y. Wang, and C. Xu, "CDX2 enhances natural killer cell-mediated immunotherapy against head and neck squamous cell carcinoma through up-regulating CXCL14," Journal of Cellular and Molecular Medicine, vol. 25, no. 10, pp. 4596-4607, 2021.

[54] T. He, M. Wang, H. Wang et al., "Evaluation of neurotensin receptor 1 as potential biomarker for prostate cancer theranostic use," European Journal of Nuclear Medicine and Molecular Imaging, vol. 46, no. 10, pp. 2199-2207, 2019.

[55] J. T. Kim, H. L. Weiss, and B. M. Evers, "Diverse expression patterns and tumorigenic role of neurotensin signaling components in colorectal cancer cells," International Journal of Oncology, vol. 50, no. 6, pp. 2200-2206, 2017.

[56] K. Takahashi, S. Ehata, K. Miyauchi, Y. Morishita, K. Miyazawa, and K. Miyazono, "Neurotensin receptor 1 signaling promotes pancreatic cancer progression," Molecular oncology, vol. 15, no. 1, pp. 151-166, 2021.

[57] Z. Wu, A. Galmiche, J. Liu et al., "Neurotensin regulation induces overexpression and activation of EGFR in HCC and restores response to erlotinib and sorafenib," Cancer Letters, vol. 388, pp. 73-84, 2017.

[58] H. Xiao, J. Wang, W. Yan et al., "GLUT1 regulates cell glycolysis and proliferation in prostate cancer," The Prostate, vol. 78, no. 2, pp. 86-94, 2018.

[59] K.-W. Min, D.-H. Kim, B. K. Son et al., "High SLC2A1 expression associated with suppressing CD8 T cells and B cells promoted cancer survival in gastric cancer," PLoS One, vol. 16, no. 3, Article ID e0245075, 2021.

[60] Z. Mo, L. Yu, Z. Cao, H. Hu, S. Luo, and S. Zhang, "Identification of a hypoxia-associated signature for lung adenocarcinoma," Frontiers in Genetics, vol. 11, p. 647, 2020.

[61] R. Sun, C. Ma, W. Wang, and S. Yang, "Upregulation of desmoglein 2 and its clinical value in lung adenocarcinoma: a comprehensive analysis by multiple bioinformatics methods," PeerJ, vol. 8, p. e8420, 2020.

[62] X.-D. Jiao, B.-D. Qin, P. You, J. Cai, and Y.-S. Zang, "The prognostic value of TP53 and its correlation with EGFR mutation in advanced non-small cell lung cancer, an analysis based on cBioPortal data base," Lung Cancer, vol. 123, pp. 70-75, 2018.

[63] E. Steels, M. Paesmans, T. Berghmans et al., "Role of p53 as a prognostic factor for survival in lung cancer: a systematic review of the literature with a meta-analysis," European Respiratory Journal, vol. 18, no. 4, pp. 705-719, 2001.

[64] X. Ma, G. Le Teuff, B. Lacas et al., "Prognostic and predictive effect of TP53 mutations in patients with non-small cell lung cancer from adjuvant cisplatin-based therapy randomized 
trials: a LACE-bio pooled analysis," Journal of Thoracic Oncology, vol. 11, no. 6, pp. 850-861, 2016.

[65] L. Mullen, M. Mengozzi, E.-M. Hanschmann, B. Alberts, and P. Ghezzi, "How the redox state regulates immunity," Free Radical Biology and Medicine, vol. 157, pp. 3-14, 2020.

[66] O. Hamid, H. Schmidt, A. Nissan et al., "A prospective phase II trial exploring the association between tumor microenvironment biomarkers and clinical activity of ipilimumab in advanced melanoma," Journal of Translational Medicine, vol. 9, no. 1, p. 204, 2011.

[67] H. Tang, Y. Wang, L. K. Chlewicki et al., "Facilitating T cell infiltration in tumor microenvironment overcomes resistance to PD-L1 blockade," Cancer Cell, vol. 29, no. 3, pp. 285-296, 2016.

[68] S.-s. Wang, W. Liu, D. Ly, H. Xu, L. Qu, and L. Zhang, "Tumor-infiltrating B cells: their role and application in antitumor immunity in lung cancer," Cellular and Molecular Immunology, vol. 16, no. 1, pp. 6-18, 2019.

[69] C. Bourgeois, B. Rocha, and C. Tanchot, "A role for CD40 expression on CD8 + T cells in the generation of CD8 + T cell memory," Science, vol. 297, no. 5589, pp. 2060-2063, 2002.

[70] M. F. Mackey, R. J. Barth, and R. J. Noelle, "The role of CD40/ CD154 interactions in the priming, differentiation, and effector function of helper and cytotoxic T cells," Journal of Leukocyte Biology, vol. 63, no. 4, pp. 418-428, 1998. 